\title{
Natural Frequencies and Mode Shapes of Mechanically Coupled Microbeam Resonators with an Application to Micromechanical Filters
}

\author{
Bashar K. Hammad \\ Department of Mechatronics Engineering, The Hashemite University, P.O. Box 150459, Zarqa 13115, Jordan \\ Correspondence should be addressed to Bashar K. Hammad; bkhammad@vt.edu
}

Received 8 February 2013; Accepted 26 July 2013; Published 12 February 2014

Academic Editor: Jeong-Hoi Koo

Copyright (C) 2014 Bashar K. Hammad. This is an open access article distributed under the Creative Commons Attribution License, which permits unrestricted use, distribution, and reproduction in any medium, provided the original work is properly cited.

\begin{abstract}
We present a methodology to calculate analytically the mode shapes and corresponding frequencies of mechanically coupled microbeam resonators. To demonstrate the methodology, we analyze a mechanical filter composed of two beams coupled by a weak beam. The boundary-value problem (BVP) for the linear vibration problem of the coupled beams depends on the number of beams and the boundary conditions of the attachment points. This implies that the system of linear homogeneous algebraic equations becomes larger as the array of resonators becomes complicated. We suggest a method to reduce the large system of equations into a smaller system. We reduce the BVP composed of five equations and twenty boundary conditions to a set of three linear homogeneous algebraic equations for three constants and the frequencies. This methodology can be simply extended to accommodate any configuration of mechanically coupled arrays. To validate our methodology, we compare our analytical results to these obtained numerically using ANSYS. We found that the agreement is excellent. We note that the weak coupling beam splits the frequency of the single resonator into two close frequencies. In addition, the effect of the coupling beam location on the natural frequencies, and hence the filter behavior, is investigated.
\end{abstract}

\section{Introduction}

Mechanically coupled microbeam resonators have attracted attention recently in the microscale realm, especially in RF MEMS [1-4]. Mode shapes and frequencies of these resonators are commonly approximated numerically using finite-element packages, such as ANSYS, COMSOL, and Coventor $[5,6]$. To the best of our knowledge, there have been few attempts to analytically generate the natural frequencies and mode shapes of multiresonator micromechanical structures characterized by distributed-parameter systems.

In this work, we present an analytical methodology to find mode shapes and the corresponding natural frequencies that can be applied to any system of coupled resonators. This methodology provides closed-form expressions for mode shapes that are easier to handle, more robust, and accurate in further analysis of coupled-resonator systems, especially in developing reduced-order models that describe the nonlinear static and dynamic characteristics of microstructures. In addition, these expressions allow designers to obtain a deeper insight into the relationship among performance metrics and the underlying microstructure dimensions, boundary conditions, and material properties. The advantages of closed-form expressions of mode shapes are unattainable using finiteelement packages which are constrained by convergence drawbacks and usually are computationally expensive.

Vyas and Bajaj [7] and Vyas et al. [8] proposed microstructures that depend on nonlinear modal interaction between microresonators. They employed linear analysis to obtain analytically the natural frequencies and associated mode shapes and defined the parameters needed to assure $1: 2$ internal resonance in T-beam [7] and pedal-type microstructures [8].

To discuss the proposed methodology in this paper and without loss of generality, we present closed-form expressions for the natural frequencies and mode shapes of micromechanical filters made of two clamped-clamped beam resonators connected via a coupling beam. The problem formulation treats the filters as distributed-parameter systems. In previous work $[6,10]$, we used the Galerkin 


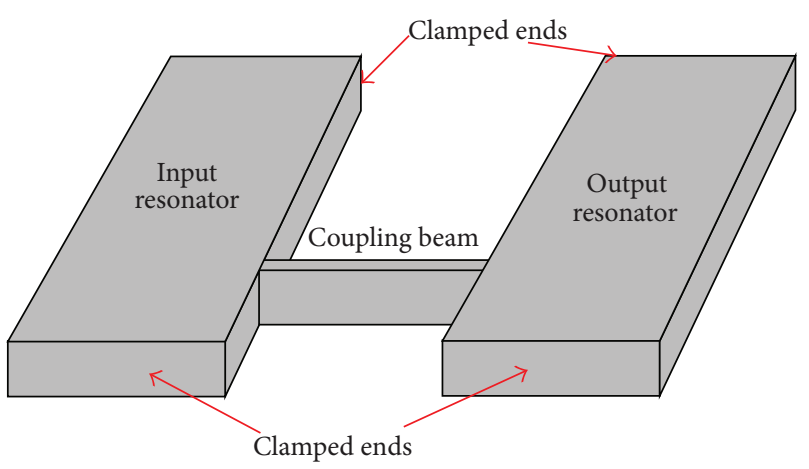

(a)

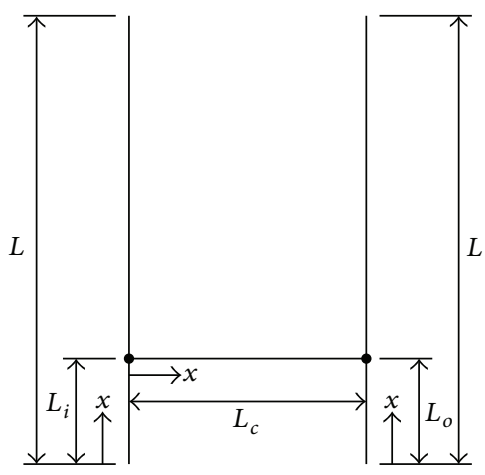

(b)

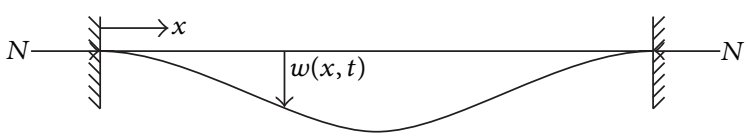

(c)

FIGURE 1: (a) A schematic drawing and (b) a schematic model of a filter made of two clamped-clamped microbeam resonators coupled by a weak beam and (c) a schematic diagram for one of the primary resonators.

procedure to develop a reduced-order model for the filter and utilized basis functions computed using the finite-element package ANSYS.

The remainder of this work is organized as follows; we derive the governing equations of the linear vibration problem in Section 2.1 and the associated boundary conditions in Section 2.2. Then, the solution of the eigenvalue problem (EVP) is discussed in Section 3.1. We manipulate the EVP to obtain a reduction in the system order in Section 3.2. The relationship between the unknowns and the mode shapes is discussed in Section 3.3 followed by a normalization scheme in Section 4. We compute the natural frequencies and mode shapes of the filter in Section 5.1, validate our results using ANSYS in Section 5.2, and compare them with these of a single clamped-clamped beam in Section 5.3. We investigate the effect of the coupling location on the natural frequency in Section 5.4 before we conclude this paper in Section 6.

\section{Problem Formulation}

We compute the natural frequencies and mode shapes of a filter composed of two clamped-clamped microbeam resonators (primary beams) coupled by a microbeam, as shown in Figure 1(a). Each primary resonator is divided into two parts at the location, where the coupling beam is attached to it, as shown in Figure 1(b). Consequently, the boundary-value problem (BVP) governing the natural frequencies and mode shapes is composed of five equations (one equation for each part of the primary beams and one for the coupling beam) and twenty boundary conditions. This problem is transformed into solving a system of twenty linear homogeneous algebraic equations for twenty constants and the natural frequencies. Using algebraic manipulations, we reduce this problem to that of solving a system of three linear homogeneous algebraic equations for three constants and the natural frequencies. The determinant of the coefficient matrix of the reduced problem yields the characteristic equation, which is solved for the natural frequencies. Then, the mode shapes are calculated.

2.1. Governing Equations. The equations of motion describing the linear, undamped, and unforced deflection of the segments of the primary beams and the coupling beam are

$E I \frac{\partial^{4} w_{i, 1}}{\partial x^{4}}+\bar{N}_{i} \frac{\partial^{2} w_{i, 1}}{\partial x^{2}}+\rho A \frac{\partial^{2} w_{i, 1}}{\partial t^{2}}=0, \quad$ where $0<x<L_{i}$,

$E I \frac{\partial^{4} w_{i, 2}}{\partial x^{4}}+\bar{N}_{i} \frac{\partial^{2} w_{i, 2}}{\partial x^{2}}+\rho A \frac{\partial^{2} w_{i, 2}}{\partial t^{2}}=0, \quad$ where $L_{i}<x<L$,

$E I \frac{\partial^{4} w_{o, 1}}{\partial x^{4}}+\overline{N_{o}} \frac{\partial^{2} w_{o, 1}}{\partial x^{2}}+\rho A \frac{\partial^{2} w_{o, 1}}{\partial t^{2}}=0, \quad$ where $0<x<L_{o}$,

$E I \frac{\partial^{4} w_{o, 2}}{\partial x^{4}}+\overline{N_{o}} \frac{\partial^{2} w_{o, 2}}{\partial x^{2}}+\rho A \frac{\partial^{2} w_{o, 2}}{\partial t^{2}}=0, \quad$ where $L_{o}<x<L$,

$E I_{c} \frac{\partial^{4} w_{c}}{\partial x^{4}}+\overline{N_{c}} \frac{\partial^{2} w_{c}}{\partial x^{2}}+\rho A_{c} \frac{\partial^{2} w_{c}}{\partial t^{2}}=0, \quad$ where $0<x<L_{c}$,

where $x$ is the position along each beam's axis, as shown in Figures $1(\mathrm{~b})$ and $1(\mathrm{c}) ; t$ is time; $w_{i, 1}$ is downward transverse deflection of the first part of input beam; $w_{i, 2}$ is downward transverse deflection of the second part of input beam; $w_{o, 1}$ is downward transverse deflection of the first part of output beam; $w_{0,2}$ is downward transverse deflection of the second part of output beam; $w_{c}$ is downward transverse deflection of the coupling beam; the deflections of all parts $w$ are functions of $x$ and $t$, as shown in Figure 1(c); $E$ is Young's modulus; $\rho$ is the material density; $I$ and $I_{c}$ are the moments of inertia of the cross sections of the primary and coupling beams, respectively; $A$ and $A_{c}$ are the areas of the cross sections of the primary and coupling beams, respectively; $L_{i}$ and $L_{o}$ are 
the positions at which the coupling beam is attached to the input and output resonators, respectively; $L$ and $L_{c}$ are the lengths of the primary and coupling beams, respectively; $\overline{N_{i}}$ is the applied compressive axial force in the input beam; $\overline{N_{o}}$ is the applied compressive axial force in the output beam; and $\overline{N_{c}}$ is the applied compressive axial force in the coupling beam. Throughout this paper, the subscripts $i, o$, and $c$ refer to quantities related to the input, output, and coupling beams, respectively. The subscripts 1 and 2 refer to the first and second parts, respectively, of each primary beam.

For convenience, we introduce the nondimensional variables:

$$
\begin{gathered}
\widehat{w}=\frac{w}{d}, \quad \hat{x}=\frac{x}{L}, \quad \widehat{t}=\frac{t}{T}, \\
\ell_{i}=\frac{L_{i}}{L}, \quad \ell_{o}=\frac{L_{o}}{L}, \quad \ell_{c}=\frac{L_{c}}{L}, \\
N_{i}=\frac{\overline{N_{i}} L^{2}}{E I}, \quad N_{o}=\frac{\overline{N_{o}} L^{2}}{E I}, \quad N_{c}=\frac{\overline{N_{c}} L^{2}}{E I_{c}},
\end{gathered}
$$

where the time is given by $T=\sqrt{\rho A L^{4} / E I}$ and $d$ is the gap between primary beams and the electrode. Substituting (2) into (1) and dropping the hats, we obtain

$$
\begin{aligned}
& \frac{\partial^{4} w_{i, 1}}{\partial x^{4}}+N_{i} \frac{\partial^{2} w_{i, 1}}{\partial x^{2}}+\frac{\partial^{2} w_{i, 1}}{\partial t^{2}}=0, \quad \text { where } 0<x<\ell_{i}, \\
& \frac{\partial^{4} w_{i, 2}}{\partial x^{4}}+N_{i} \frac{\partial^{2} w_{i, 2}}{\partial x^{2}}+\frac{\partial^{2} w_{i, 2}}{\partial t^{2}}=0, \quad \text { where } \ell_{i}<x<1, \\
& \frac{\partial^{4} w_{o, 1}}{\partial x^{4}}+N_{o} \frac{\partial^{2} w_{o, 1}}{\partial x^{2}}+\frac{\partial^{2} w_{o, 1}}{\partial t^{2}}=0, \quad \text { where } 0<x<\ell_{o}, \\
& \frac{\partial^{4} w_{o, 2}}{\partial x^{4}}+N_{o} \frac{\partial^{2} w_{o, 2}}{\partial x^{2}}+\frac{\partial^{2} w_{o, 2}}{\partial t^{2}}=0, \quad \text { where } \ell_{o}<x<1, \\
& \frac{\partial^{4} w_{c}}{\partial x^{4}}+N_{c} \frac{\partial^{2} w_{c}}{\partial x^{2}}+\left(\frac{h}{h_{c}}\right)^{2} \frac{\partial^{2} w_{c}}{\partial t^{2}}=0, \quad \text { where } 0<x<\ell_{c},
\end{aligned}
$$

where $h$ and $h_{c}$ are the thicknesses of the primary and coupling beams, respectively.

2.2. Boundary Conditions. For the clamped (fixed) ends of the primary beams, the bending moments and shear forces are unrestricted, but the deflections and the slopes vanish; that is,

$$
\begin{array}{ll}
w_{i, 1}(0)=0, & \left.\frac{\partial w_{i, 1}}{\partial x}\right|_{x=0}=0, \\
w_{o, 1}(0)=0, & \left.\frac{\partial w_{o, 1}}{\partial x}\right|_{x=0}=0, \\
w_{i, 2}(1)=0, & \left.\frac{\partial w_{i, 2}}{\partial x}\right|_{x=1}=0 \\
w_{o, 2}(1)=0, & \left.\frac{\partial w_{o, 2}}{\partial x}\right|_{x=1}=0 .
\end{array}
$$

At the attachment point in each of the primary beams, the deflection, slope, and moment are continuous. Hence, we have

$$
\begin{gathered}
w_{i, 1}\left(\ell_{i}\right)=w_{i, 2}\left(\ell_{i}\right),\left.\quad \frac{\partial w_{i, 1}}{\partial x}\right|_{x=\ell_{i}}=\left.\frac{\partial w_{i, 2}}{\partial x}\right|_{x=\ell_{i}}, \\
\left.\frac{\partial^{2} w_{i, 1}}{\partial x^{2}}\right|_{x=\ell_{i}}=\left.\frac{\partial^{2} w_{i, 2}}{\partial x^{2}}\right|_{x=\ell_{i}}, \\
w_{o, 1}\left(\ell_{o}\right)=w_{o, 2}\left(\ell_{o}\right),\left.\quad \frac{\partial w_{o, 1}}{\partial x}\right|_{x=\ell_{o}}=\left.\frac{\partial w_{o, 2}}{\partial x}\right|_{x=\ell_{o}}, \\
\left.\frac{\partial^{2} w_{o, 1}}{\partial x^{2}}\right|_{x=\ell_{o}}=\left.\frac{\partial^{2} w_{o, 2}}{\partial x^{2}}\right|_{x=\ell_{o}} .
\end{gathered}
$$

The deflections of the coupling beam are the same as the deflections of the primary beams at the attachment points, and the slopes of the coupling beam at these attachment points vanish. Therefore,

$$
\begin{array}{cc}
w_{c}(0)=w_{i, 1}\left(\ell_{i}\right), & w_{c}\left(\ell_{c}\right)=w_{o, 1}\left(\ell_{o}\right), \\
\left.\frac{\partial w_{c}}{\partial x}\right|_{x=0}=0, & \left.\frac{\partial w_{c}}{\partial x}\right|_{x=\ell_{c}}=0 .
\end{array}
$$

The shear forces at the ends of the coupling beam are equal to the changes in the shear forces in the primary beams. These conditions yield

$$
\begin{aligned}
& \left.\frac{\partial^{3} w_{i, 1}}{\partial x^{3}}\right|_{x=\ell_{i}}-\left.\frac{\partial^{3} w_{i, 2}}{\partial x^{3}}\right|_{x=\ell_{i}}=\left.\frac{I_{c}}{I} \frac{\partial^{3} w_{c}}{\partial x^{3}}\right|_{x=0}, \\
& \left.\frac{\partial^{3} w_{o, 1}}{\partial x^{3}}\right|_{x=\ell_{o}}-\left.\frac{\partial^{3} w_{o, 2}}{\partial x^{3}}\right|_{x=\ell_{o}}=-\left.\frac{I_{c}}{I} \frac{\partial^{3} w_{c}}{\partial x^{3}}\right|_{x=\ell_{c}} .
\end{aligned}
$$

\section{Eigenvalue Problem}

3.1. Natural Frequencies of the Filter. We assume that solutions of the equations of motion, (3), consist of spatial and temporal parts given as follows:

$$
\begin{array}{cc}
w_{i, 1}(x, t)=\phi_{i, 1}(x) \exp (j \omega t), & \text { where } 0<x<\ell_{i}, \\
w_{i, 2}(x, t)=\phi_{i, 2}(x) \exp (j \omega t), & \text { where } \ell_{i}<x<1, \\
w_{o, 1}(x, t)=\phi_{o, 1}(x) \exp (j \omega t), & \text { where } 0<x<\ell_{o}, \\
w_{o, 2}(x, t)=\phi_{o, 2}(x) \exp (j \omega t), & \text { where } \ell_{o}<x<1, \\
w_{c}(x, t)=\phi_{c}(x) \exp (j \omega t), & \text { where } 0<x<\ell_{c} .
\end{array}
$$

The $\phi_{i, 1}$ is the mode shape of the first part of input beam, $\phi_{i, 2}$ is the mode shape of the second part of input beam, $\phi_{o, 1}$ is the mode shape of the first part of output beam, $\phi_{o, 2}$ is the mode shape of the second part of output beam, $\phi_{c}$ is the mode shape of the coupling beam, and the $\omega$ 's are the nondimensional 
natural frequencies corresponding to these mode shapes. Substituting (8) into (3), we obtain

$$
\begin{array}{cc}
\phi_{i, 1}^{i v}(x)+N_{i} \phi_{i, 1}^{\prime \prime}(x)-\omega^{2} \phi_{i, 1}(x)=0, & \text { where } 0<x<\ell_{i}, \\
\phi_{i, 2}^{i v}(x)+N_{i} \phi_{i, 2}^{\prime \prime}(x)-\omega^{2} \phi_{i, 2}(x)=0, & \text { where } \ell_{i}<x<1, \\
\phi_{o, 1}^{i v}(x)+N_{o} \phi_{o, 1}^{\prime \prime}(x)-\omega^{2} \phi_{o, 1}(x)=0, & \text { where } 0<x<\ell_{o}, \\
\phi_{o, 2}^{i v}(x)+N_{o} \phi_{o, 2}^{\prime \prime}(x)-\omega^{2} \phi_{o, 2}(x)=0, & \text { where } \ell_{o}<x<1, \\
\phi_{c}^{i v}(x)+N_{c} \phi_{c}^{\prime \prime}(x)-\left(\frac{h}{h_{c}}\right)^{2} \omega^{2} \phi_{c}(x)=0, & \text { where } 0<x<\ell_{c} .
\end{array}
$$

Substituting (8) into the boundary conditions, as shown in Section 2.2, yields the following.

(i) For the clamped edges:

$$
\begin{array}{ll}
\phi_{i, 1}(0)=0, & \phi_{i, 1}^{\prime}(0)=0, \\
\phi_{o, 1}(0)=0, & \phi_{o, 1}^{\prime}(0)=0, \\
\phi_{i, 2}(1)=0, & \phi_{i, 2}^{\prime}(1)=0, \\
\phi_{o, 2}(1)=0, & \phi_{o, 2}^{\prime}(1)=0 .
\end{array}
$$

(ii) At the attachment points in the primary beams:

$$
\begin{gathered}
\phi_{i, 1}\left(\ell_{i}\right)=\phi_{i, 2}\left(\ell_{i}\right), \quad \phi_{i, 1}^{\prime}\left(\ell_{i}\right)=\phi_{i, 2}^{\prime}\left(\ell_{i}\right), \\
\phi_{i, 1}^{\prime \prime}\left(\ell_{i}\right)=\phi_{i, 2}^{\prime \prime}\left(\ell_{i}\right), \\
\phi_{o, 1}\left(\ell_{o}\right)=\phi_{o, 2}\left(\ell_{o}\right), \quad \phi_{o, 1}^{\prime}\left(\ell_{o}\right)=\phi_{o, 2}^{\prime}\left(\ell_{o}\right), \\
\phi_{o, 1}^{\prime \prime}\left(\ell_{o}\right)=\phi_{o, 2}^{\prime \prime}\left(\ell_{o}\right) .
\end{gathered}
$$

(iii) At the attachment points in the coupling beam:

$$
\begin{array}{cl}
\phi_{c}(0)=\phi_{i, 1}\left(\ell_{i}\right), & \phi_{c}\left(\ell_{c}\right)=\phi_{o, 1}\left(\ell_{o}\right), \\
\phi_{c}^{\prime}(0)=0, \quad \phi_{c}^{\prime}\left(\ell_{c}\right)=0 .
\end{array}
$$

(iv) The shear force at the attachment points:

$$
\begin{aligned}
\phi_{i, 1}^{\prime \prime \prime}\left(\ell_{i}\right)-\phi_{i, 2}^{\prime \prime \prime}\left(\ell_{i}\right) & =\frac{I_{c}}{I} \phi_{c}^{\prime \prime \prime}(0), \\
\phi_{o, 1}^{\prime \prime \prime}\left(\ell_{o}\right)-\phi_{o, 2}^{\prime \prime \prime}\left(\ell_{o}\right) & =-\frac{I_{c}}{I} \phi_{c}^{\prime \prime \prime}\left(\ell_{c}\right) .
\end{aligned}
$$

Assuming a solution to (9) of the form $a \exp (\sigma x)$ yields the general solution

$$
\begin{aligned}
\phi_{i, 1}(x)= & C_{1} \cos \left[\beta_{i 1} x\right]+C_{2} \sin \left[\beta_{i 1} x\right]+C_{1 a} \cosh \left[\beta_{i 2} x\right] \\
& +C_{2 a} \sinh \left[\beta_{i 2} x\right], \quad \text { where } 0<x<\ell_{i}, \\
\phi_{i, 2}(x)= & C_{3} \cos \left[\beta_{i 1}(1-x)\right]+C_{4} \sin \left[\beta_{i 1}(1-x)\right] \\
& +C_{3 a} \cosh \left[\beta_{i 2}(1-x)\right]+C_{4 a} \sinh \left[\beta_{i 2}(1-x)\right], \\
& \text { where } \ell_{i}<x<1, \\
\phi_{o, 1}(x)= & C_{5} \cos \left[\beta_{o 1} x\right]+C_{6} \sin \left[\beta_{o 1} x\right]+C_{5 a} \cosh \left[\beta_{o 2} x\right] \\
& +C_{6 a} \sinh \left[\beta_{o 2} x\right], \quad \text { where } 0<x<\ell_{o}, \\
\phi_{o, 2}(x)= & C_{7} \cos \left[\beta_{o 1}(1-x)\right]+C_{8} \sin \left[\beta_{o 1}(1-x)\right] \\
& +C_{7 a} \cosh \left[\beta_{o 2}(1-x)\right]+C_{8 a} \sinh \left[\beta_{o 2}(1-x)\right], \\
\quad & \text { where } \ell_{o}<x<1, \\
\phi_{c}(x)= & C_{9} \cos \left[\beta_{c 1} x\right]+C_{10} \sin \left[\beta_{c 1} x\right]+C_{11} \cosh \left[\beta_{c 2} x\right] \\
& +C_{12} \sinh \left[\beta_{c 2} x\right], \quad \text { where } 0<x<\ell_{c} .
\end{aligned}
$$

The constants $\beta$ 's are given by

$$
\begin{aligned}
& \beta_{i 1}=\sqrt{+\frac{N_{i}}{2}+\frac{1}{2} \sqrt{N_{i}^{2}+4 \omega^{2}},} \\
& \beta_{i 2}=\sqrt{-\frac{N_{i}}{2}+\frac{1}{2} \sqrt{N_{i}^{2}+4 \omega^{2}}},
\end{aligned}
$$

$$
\beta_{o 1}=\sqrt{+\frac{N_{o}}{2}+\frac{1}{2} \sqrt{N_{o}^{2}+4 \omega^{2}}},
$$$$
\beta_{o 2}=\sqrt{-\frac{N_{o}}{2}+\frac{1}{2} \sqrt{N_{o}^{2}+4 \omega^{2}}},
$$

$$
\beta_{c 1}=\sqrt{+\frac{N_{c}}{2}+\frac{1}{2} \sqrt{N_{c}^{2}+4\left(\frac{h}{h_{c}}\right)^{2} \omega^{2}},}
$$

$$
\beta_{c 2}=\sqrt{-\frac{N_{c}}{2}+\frac{1}{2} \sqrt{N_{c}^{2}+4\left(\frac{h}{h_{c}}\right)^{2} \omega^{2}} .}
$$

Substituting (17) into (10)-(16) leads to twenty linear homogeneous algebraic equations in the twenty unknown coefficients $C_{1}, C_{2}, \ldots, C_{12}$ and $C_{1 a}, C_{2 a}, \ldots, C_{8 a}$, which can be written in matrix form as

$$
\mathrm{MC}=\mathbf{0},
$$

where $\mathbf{M}$ is a $20 \times 20$ matrix, $\mathbf{C}$ is a $20 \times 1$ vector whose elements are the above unknown coefficients, and $\mathbf{0}$ is a $20 \times 1$ zero vector. The elements of $\mathbf{M}$ are functions of $\beta$, and hence $\omega$, and the dimensions of the filter. 
3.2. Reduction of the Eigenvalue Problem. Equation (19) has nontrivial solutions $\mathbf{C}$ if and only if the coefficient matrix $\mathbf{M}$ is singular; that is, the determinant of the $20 \times 20$ matrix $\mathbf{M}$ is zero. To reduce the cost of generating this determinant and, more importantly, obtain a deeper insight into the relationship among the unknowns, we analytically manipulate the governing equation (19). To this end, we substitute (17) into (10), solve the $C_{j a}$ in terms of the $C_{j}$, and rewrite (17) as

$$
\begin{aligned}
& \phi_{i, 1}(x)=C_{1}\left\{\cos \left[\beta_{i 1} x\right]-\cosh \left[\beta_{i 2} x\right]\right\} \\
& +C_{2}\left\{\sin \left[\beta_{i 1} x\right]-\sinh \left[\beta_{i 2} x\right]\right\}, \\
& \text { where } 0<x<\ell_{i} \text {, } \\
& \phi_{i, 2}(x)=C_{3}\left\{\cos \left[\beta_{i 1}(1-x)\right]-\cosh \left[\beta_{i 2}(1-x)\right]\right\} \\
& +C_{4}\left\{\sin \left[\beta_{i 1}(1-x)\right]-\sinh \left[\beta_{i 2}(1-x)\right]\right\}, \\
& \text { where } \ell_{i}<x<1 \text {, } \\
& \phi_{o, 1}(x)=C_{5}\left\{\cos \left[\beta_{o 1} x\right]-\cosh \left[\beta_{o 2} x\right]\right\} \\
& +C_{6}\left\{\sin \left[\beta_{o 1} x\right]-\sinh \left[\beta_{o 2} x\right]\right\}, \\
& \text { where } 0<x<\ell_{o} \text {, } \\
& \phi_{o, 2}(x)=C_{7}\left\{\cos \left[\beta_{o 1}(1-x)\right]-\cosh \left[\beta_{o 2}(1-x)\right]\right\} \\
& +C_{8}\left\{\sin \left[\beta_{o 1}(1-x)\right]-\sinh \left[\beta_{o 2}(1-x)\right]\right\}, \\
& \text { where } \ell_{o}<x<1 \text {, } \\
& \phi_{c}(x)=C_{9} \cos \left[\beta_{c 1} x\right]+C_{10} \sin \left[\beta_{c 1} x\right] \\
& +C_{11} \cosh \left[\beta_{c 2} x\right]+C_{12} \sinh \left[\beta_{c 2} x\right], \\
& \text { where } 0<x<\ell_{c} \text {. }
\end{aligned}
$$

We substitute (20)-(24) into (11), (12), (14), and (15) and solve the resulting nine equations for the $C_{j}$ in terms of $C_{1}, C_{5}$, and $C_{9}$, which represent the input, output, and coupling beams, respectively. Then, using the remaining three boundary conditions, (13) and (16), we obtain the reduced problem

$$
\mathrm{M}_{\mathbf{r}} \mathrm{C}_{\mathbf{r}}=\mathbf{0}
$$

where

$$
\mathbf{M}_{\mathbf{r}}=\left[\begin{array}{lll}
g_{11} & g_{12} & g_{13} \\
g_{21} & g_{22} & g_{23} \\
g_{31} & g_{32} & g_{33}
\end{array}\right], \quad \mathbf{C}_{\mathbf{r}}=\left(\begin{array}{l}
C_{1} \\
C_{5} \\
C_{9}
\end{array}\right)
$$

The zero vector $\mathbf{0}$ in this case is a $3 \times 1$ vector and the elements $g_{m n}$ are functions of the $\beta$ 's, which are given in (18). Setting the determinant of $\mathbf{M}_{\mathbf{r}}$ equal to zero yields the characteristic equation

$$
\operatorname{Det}\left[\mathbf{M}_{\mathbf{r}}\right]=0
$$

which is solved for the natural frequencies $\omega_{n}$ of the filter.
3.3. Mode Shapes of the Filter. Associated with each $\omega_{n}$ satisfying (27) is a mode shape. To compute this mode shape, we substitute $\omega_{n}$ into (18), obtain numerical values for the $\beta$ 's, and substitute these $\beta$ 's into (20)-(24). Then, we use the boundary conditions to find numerical relations among the unknowns and end up with

$$
a_{1} C_{1}+a_{2} C_{5}=0 \text {. }
$$

Depending on $\omega_{n}$, one of the following two cases for the values of the constants $a_{1}$ and $a_{2}$ is obtained:

Case 1: $a_{1}=a_{2} \Rightarrow C_{1}=-C_{5} \Rightarrow$ primary beams vibrate out-of-phase.

Case 2: $a_{1}=-a_{2} \Rightarrow C_{1}=C_{5} \Rightarrow$ primary beams vibrate in-phase.

By setting $C_{1}$ equal to one, we find numerical values for all of the $C$ 's in (20)-(24) and hence the mode shape corresponding to $\omega_{n}$.

\section{Normalization of Mode Shapes}

Because the algebraic system of equations, (19), is homogeneous, it follows that if the vector $\mathbf{C}$ is a solution of the equation, then $\alpha \mathbf{C}$ is also a solution, where $\alpha$ is an arbitrary constant. This implies that the mode shapes are unique within a constant. We normalize the mode shapes (i.e., render them unique) by setting [11]

$$
\int_{x_{1}}^{x_{2}}(\alpha \phi(x))^{2} \mathrm{~d} x=1,
$$

where $\phi(x)$ is the mode shape. Hence, the constant $\alpha$ is given by

$$
\alpha=\frac{1}{\sqrt{\int_{x_{1}}^{x_{2}} \phi(x)^{2} \mathrm{~d} x}} .
$$

Considering the topology of the filter structure investigated in this work and the analysis of the reduced-order model performed in [6], we find that the constant $\alpha_{r}$ associated with $\phi_{r}$ and $\omega_{r}$ is given by

$$
\begin{gathered}
\alpha_{r}=\left(\int_{0}^{\ell_{i}} \phi_{i, 1}^{2}(x) \mathrm{d} x+\int_{\ell_{i}}^{1} \phi_{i, 2}^{2}(x) \mathrm{d} x+\int_{0}^{\ell_{o}} \phi_{o, 1}^{2}(x) \mathrm{d} x\right. \\
\left.+\int_{\ell_{o}}^{1} \phi_{o, 2}^{2}(x) \mathrm{d} x+T_{c}^{2} \int_{0}^{\ell_{c}} \phi_{c}^{2}(x) \mathrm{d} x\right)^{-1 / 2} .
\end{gathered}
$$

The parameter $T_{c}^{2}=A_{c} / A_{p}$ is the ratio of the cross section area of the coupling beam to that of the primary beam.

\section{Results and Discussion}

We utilize the developed methodology to study the filter fabricated and tested by Bannon et al. [9] but without considering the frequency modification factor or any adjustments due to fabrication processes. The design parameters, dimensions, and material properties of the filter needed in this analysis are obtained from [9] and listed in Table 1. 
5.1. Closed-Form Expression of the Mode Shapes. In this section, we employ the methodology discussed in the preceding sections to present closed-form expressions for the mode shapes. To avoid unnecessary repetition, we show here details of the first mode shape for the filter specified in Table 1. In this analysis, because the fabrication process involves a relatively long-time (one hour) annealing of the structure [9], we assume that all of the beams are free of residual stresses; that is, $N_{i}=N_{o}=N_{c}=0$.

The first nondimensional natural frequency of the filter (i.e., the smallest solution of the characteristic equation (27)) is $\omega_{1}=22.363(=9.471 \mathrm{MHz})$. Substituting this frequency into the boundary conditions, we end up with $C_{1}=C_{5}$. By setting $C_{1}$ equal to one, we find numerical values for all of the $C$ 's in (20)-(24). Using the normalization condition given in (31), we obtain $\alpha_{1}=0.710$ and, hence, the following normalized closed-form expression for the first global mode shape of the filter:

$$
\begin{aligned}
& \phi_{i, 1}(x)=0.710\{\cos [4.729 x]-\cosh [4.729 x]\} \\
& -0.702\{\sin [4.729 x]-\sinh [4.729 x]\}, \\
& \text { where } 0<x<\ell_{i} \text {, } \\
& \phi_{i, 2}(x)=0.707\{\cos [4.729(1-x)]-\cosh [4.729(1-x)]\} \\
& -0.695\{\sin [4.729(1-x)]-\sinh [4.729(1-x)]\}, \\
& \text { where } \ell_{i}<x<1 \text {, } \\
& \phi_{o, 1}(x)=0.710\{\cos [4.729 x]-\cosh [4.729 x]\} \\
& -0.702\{\sin [4.729 x]-\sinh [4.729 x]\}, \\
& \text { where } 0<x<\ell_{0} \text {, } \\
& \phi_{o, 2}(x)=0.707\{\cos [4.729(1-x)]-\cosh [4.729(1-x)]\} \\
& -0.695\{\sin [4.729(1-x)]-\sinh [4.729(1-x)]\}, \\
& \text { where } \ell_{o}<x<1 \text {, } \\
& \phi_{c}(x)=-0.034 \cos [4.729 x]-0.082 \sin [4.729 x] \\
& -0.100 \cosh [4.729 x]+0.082 \sinh [4.729 x] \text {, } \\
& \text { where } 0<x<\ell_{c} \text {. }
\end{aligned}
$$

The normalized expression of the first mode shape of the filter, (32), is shown in Figure 2. In all mode shapes shown in this paper, we use the normalization scheme in the same way discussed above. In addition, in all mode shape figures, red lines are obtained from (20) and (22), blue lines are obtained from from (21) and (23), and green lines are obtained from from (24).

5.2. Validation of the Results. We use the finite-element software ANSYS and the structural elements BEAM3 and BEAM4 to validate our model and methodology. The degrees of freedom of elements are constrained such that only the transverse motion is allowed. Using modal analysis solution,
TABLE 1: Filter specifications [9].

\begin{tabular}{lc}
\hline Parameter & Design value \\
\hline Primary resonator length, $L(\mu \mathrm{m})$ & 40.8 \\
Primary resonator width, $b(\mu \mathrm{m})$ & 8.0 \\
Coupling location, $L_{i}$ and $L_{o}(\mu \mathrm{m})$ & 4.08 \\
Coupling beam length, $L_{c}(\mu \mathrm{m})$ & 20.35 \\
Coupling beam width, $b_{c}(\mu \mathrm{m})$ & 0.75 \\
Structural thickness, $h(\mu \mathrm{m})$ & 1.9 \\
Young's modulus, $E(\mathrm{GPa})$ & 150 \\
Polysilicon density, $\rho\left(\mathrm{kg} / \mathrm{m}^{3}\right)$ & 2,300 \\
\hline
\end{tabular}

we obtained natural frequencies of the filter structure and listed them in Table 2. Inspecting the results obtained from the analytical approach discussed in this paper and the numerical approach from ANSYS reveals that the agreement between them is excellent. In addition, the mode shapes of the filter structure obtained from both of the approaches (being in-phase or out-of-phase, and their order as illustrated in the next section) are in complete agreement.

5.3. Relation of the Mode Shapes of the Filter to Those of Single Clamped-Clamped Resonators. In Table 2, we list the lowest 10 natural frequencies of the filter, that is, the lowest 10 solutions of the characteristic equation, (27), considering the specifications listed in Table 1 and assuming that the structure is free from any residual stresses. Due to the effect of the weak coupling beam shown in Figure 1(a), the natural frequencies of the single resonator are split into two close frequencies for the filter, as listed in Table 2: one frequency corresponds to an in-phase mode and the other corresponds to an out-ofphase mode. In filter terminology, the in-phase mode is called a symmetric mode and the out-of-phase mode is called an antisymmetric mode. Next, we discuss in more detail these mode shapes.

Figures 2 and 3 show the first and second global mode shapes of the filter, respectively. The input and output resonators oscillate in the first mode of a single clampedclamped beam resonator. However, they oscillate in-phase in the first mode of the filter, whereas they oscillate $180^{\circ}$ outof-phase in the second mode of the filter. The frequencies of the first and second modes of the filter are shifted to slightly smaller and larger values, respectively, compared to the frequency of the first mode of a single clamped-clamped resonator, as shown in Table 2.

The third and fourth mode shapes of the filter are shown in Figures 4 and 5, respectively. The input and output resonators oscillate in the second mode of a single clampedclamped resonator. They oscillate in-phase in the third mode and out-of-phase in the fourth mode. The sixth and seventh mode shapes of the filter are shown in Figures 7 and 8, respectively. The input and output resonators oscillate in the third mode of a single clamped-clamped resonator, but they oscillate out-of-phase in the sixth mode and in-phase in the seventh mode of the filter. It follows from the eighth and ninth mode shapes of the filter, shown in Figures 9 and 10, that 
TABLE 2: Natural frequencies (in $\mathrm{MHz}$ ) for a filter and a single resonator identical to the resonators of the filter.

\begin{tabular}{|c|c|c|c|c|c|}
\hline \multirow{3}{*}{ Mode shape number } & \multicolumn{2}{|c|}{ Filter } & & \multicolumn{2}{|c|}{ Single resonator } \\
\hline & \multicolumn{3}{|c|}{ Natural frequency } & \multirow{2}{*}{ Mode shape number } & \multirow{2}{*}{ Natural frequency } \\
\hline & Analytical & ANSYS & Difference (\%) & & \\
\hline 1 & 9.471 & 9.460 & 0.116 & \multirow{2}{*}{1} & \multirow{2}{*}{9.475} \\
\hline 2 & 9.479 & 9.469 & 0.105 & & \\
\hline 3 & 26.015 & 25.909 & 0.407 & \multirow{2}{*}{2} & \multirow{2}{*}{26.118} \\
\hline 4 & 26.099 & 25.991 & 0.414 & & \\
\hline 5 & 37.787 & 37.622 & 0.437 & - & - \\
\hline 6 & 51.017 & 50.570 & 0.876 & \multirow{2}{*}{3} & \multirow{2}{*}{51.202} \\
\hline 7 & 51.385 & 50.930 & 0.885 & & \\
\hline 8 & 83.574 & 82.345 & 1.470 & \multirow{2}{*}{4} & \multirow{2}{*}{84.639} \\
\hline 9 & 84.453 & 83.176 & 1.512 & & \\
\hline 10 & 104.463 & 102.740 & 1.649 & - & - \\
\hline
\end{tabular}

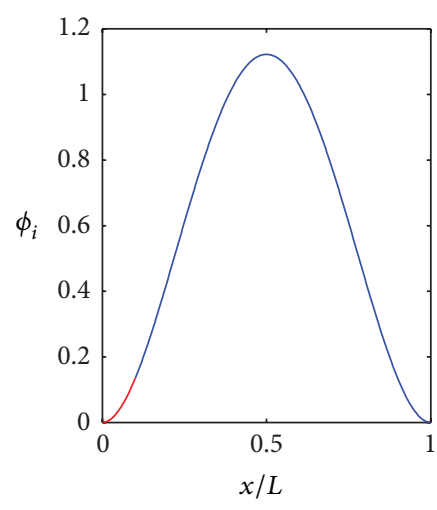

(a)

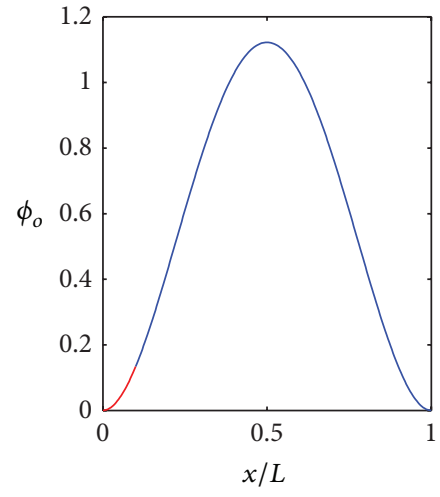

(b)

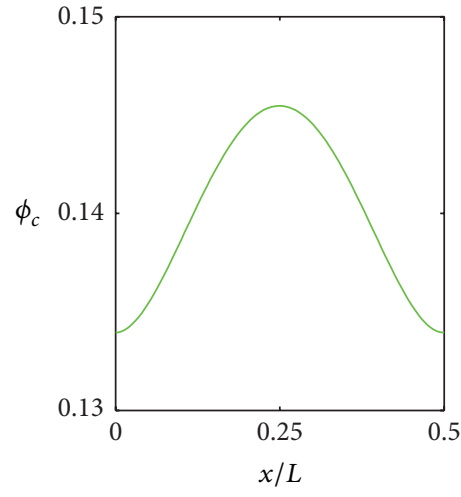

(c)

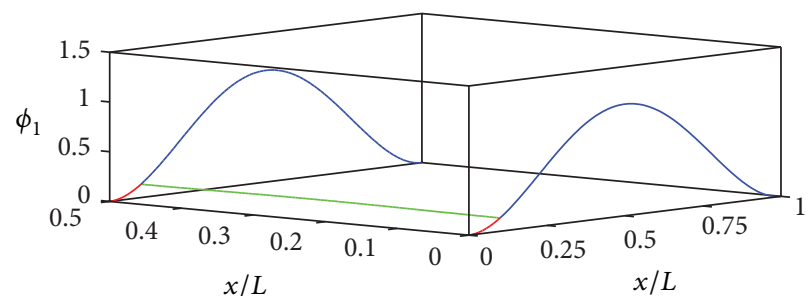

(d)

FIGURE 2: First mode shape of the filter: (a) input, (b) output, (c) coupling beams, and (d) combination of (a), (b), and (c).

the filter oscillates in the fourth mode of a single clampedclamped beam.

Inspecting Table 2, we note that, when the resonators of the filter oscillate in a symmetric mode of a single clampedclamped beam, the single beam frequency is split into two frequencies: one slightly higher and the other slightly lower than that of the single resonator. In contrast, when the primary resonators vibrate in an antisymmetric mode of a single beam, both frequencies of the filter are slightly smaller than the corresponding frequency of the single beam.

For the dimensions listed in Table 1, we note that, after every four modes, there is a mode in which the vibrations of the primary beams (input and output beams) are very small compared to the vibration of the coupling beam. It follows from Figures 6 and 11 that, in the fifth and tenth modes of the filter, the coupling beam oscillates in the first and second modes, respectively, of a single beam with slightly flexible clamping points (the rigidity of the clamping points is finite). We observe that these modes appear at relatively larger frequencies because our model considers transverse vibrations of the beams; that is, the vibration is in the direction perpendicular to the plane that contains the input, output, and coupling beams. However, because the flexural stiffness EI of the coupling beam in this paper in the inplane direction is about six times smaller than its stiffness in the transverse direction, the in-plane modes have relatively small frequencies. However, due to the way these types of filters are actuated (see [6]), the in-plane modes will not be activated, thereby justifying the use of a transverse-vibration model only. 


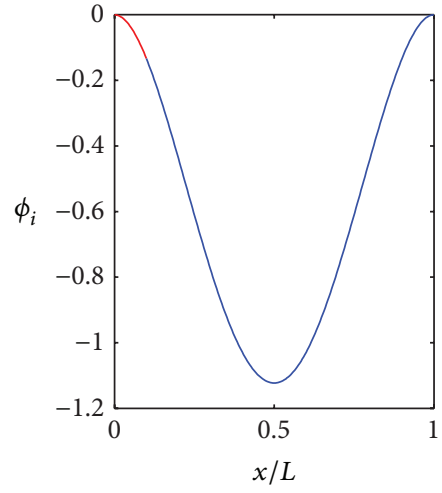

(a)

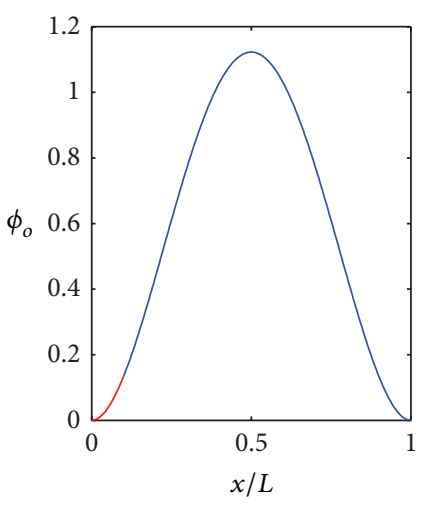

(b)

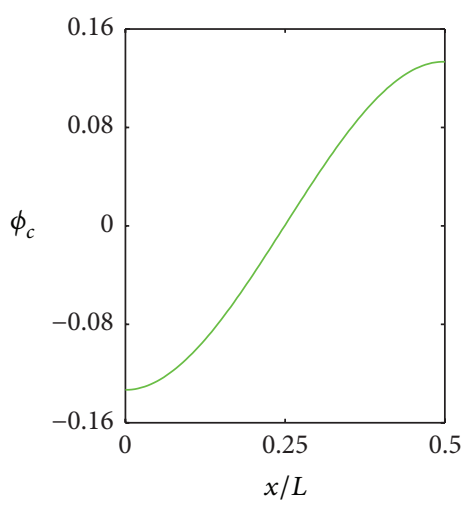

(c)

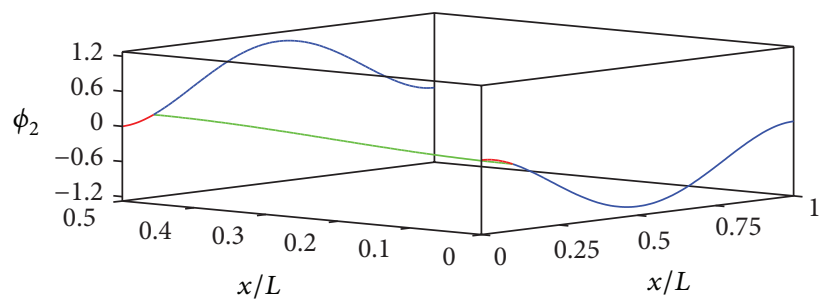

(d)

Figure 3: Second mode shape of the filter: (a) input, (b) output, (c) coupling beams, and (d) combination of (a), (b), and (c).

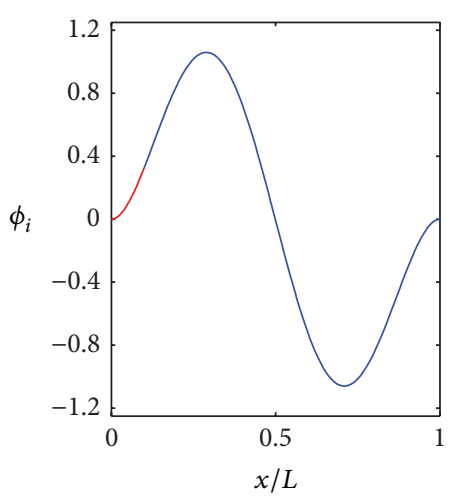

(a)

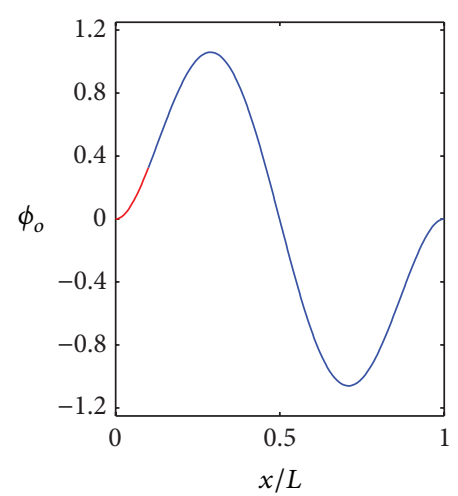

(b)

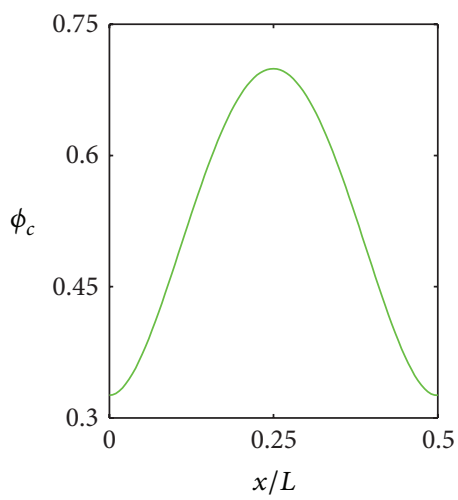

(c)

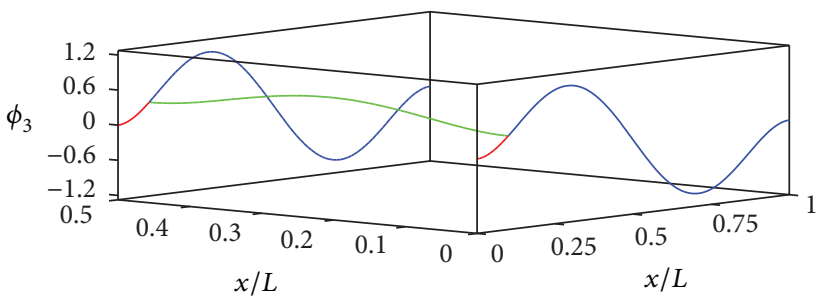

(d)

Figure 4: Third mode shape of the filter: (a) input, (b) output, (c) coupling beams, and (d) combination of (a), (b), and (c).

5.4. Coupling Location. In this section, we study the effect of changing the attachment location of the coupling beam to the primary resonators on the first and second natural frequencies of the filter, and, to be more specific, on their average and difference. The average and difference of the first and second natural frequencies are indicators of the actual center frequency and bandwidth, respectively, of the filter when an electric signal is applied to the input and output resonators. In this section, we indicate the center frequency and bandwidth of the unactuated filter by $F_{0}$ and $\mathrm{BW}_{0}$, respectively. We focus on the first and second frequencies because it is common to excite directly the filter with a frequency in the neighborhood 


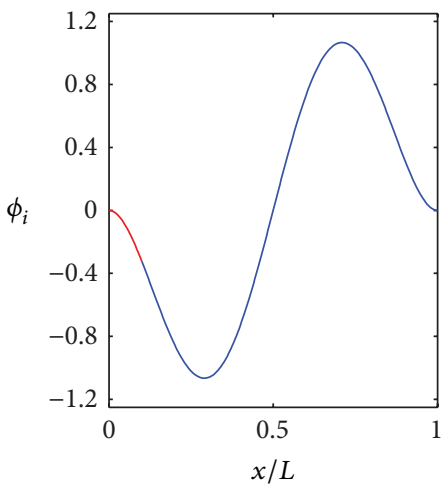

(a)

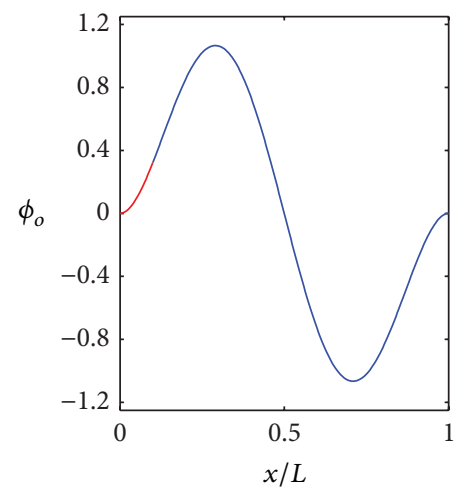

(b)

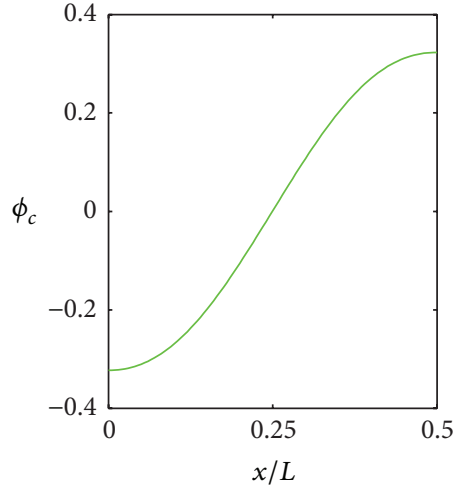

(c)

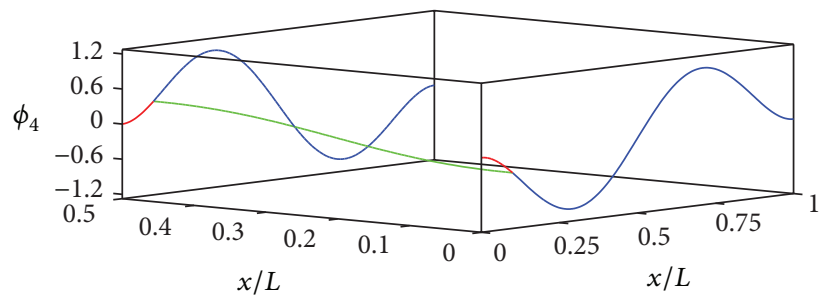

(d)

FigurE 5: Fourth mode shape of the filter: (a) input, (b) output, (c) coupling beams, and (d) combination of (a), (b), and (c).

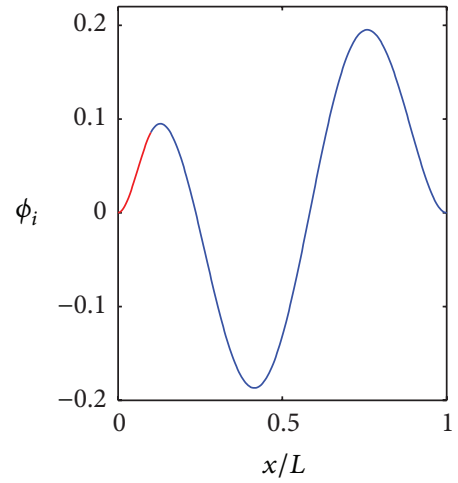

(a)

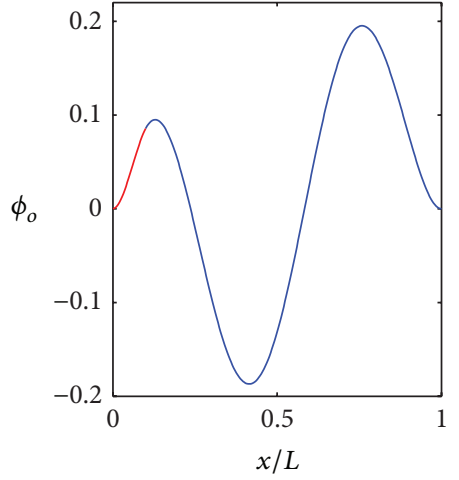

(b)

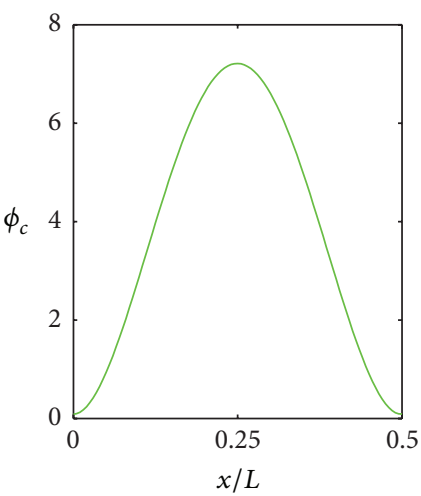

(c)

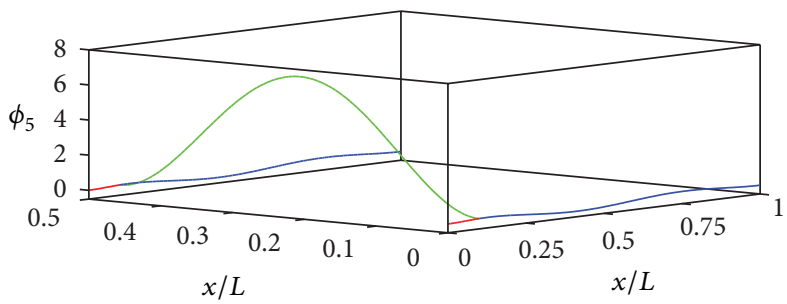

(d)

FIGURE 6: Fifth mode shape of the filter: (a) input, (b) output, (c) coupling beams, and (d) combination of (a), (b), and (c).

of these frequencies. In case higher modes are excited directly, the center frequency and bandwidth of the filter will change. Figure 12 shows variations of the first and second natural frequencies of the filter with the attachment location. When the coupling beam is attached to the clamping points of the primary beams, the frequencies of the filter are the same as the frequencies of a single resonator. This is expected because in this case the two primary resonators are not actually coupled. As the attachment location moves away from the clamping point towards the middle of the primary beams, the first natural frequency decreases and the second natural frequency increases until they reach their extrema 


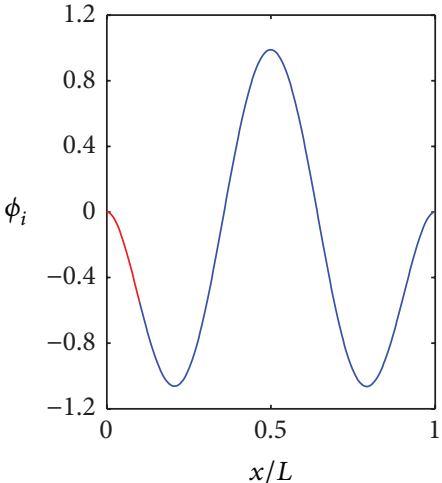

(a)

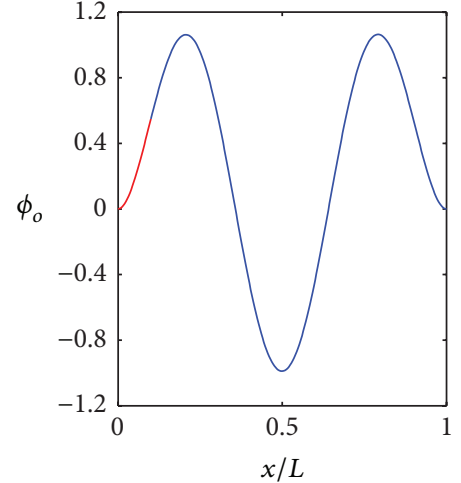

(b)

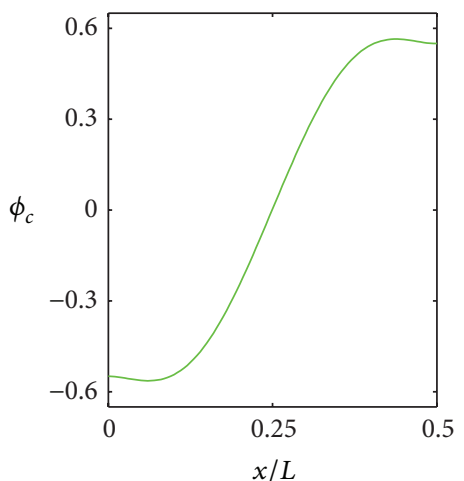

(c)

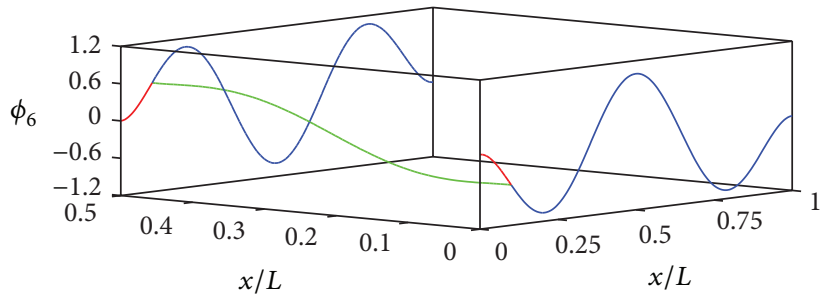

(d)

Figure 7: Sixth mode shape of the filter: (a) input, (b) output, (c) coupling beams, and (d) combination of (a), (b), and (c).

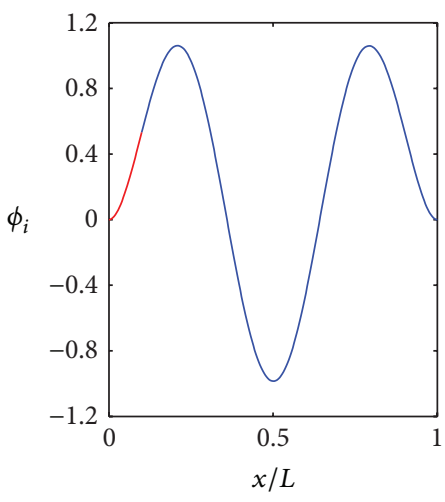

(a)

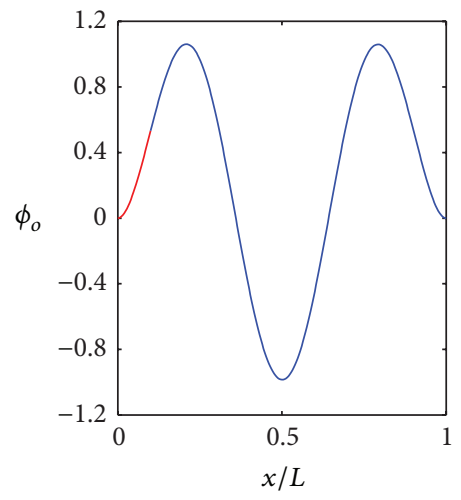

(b)

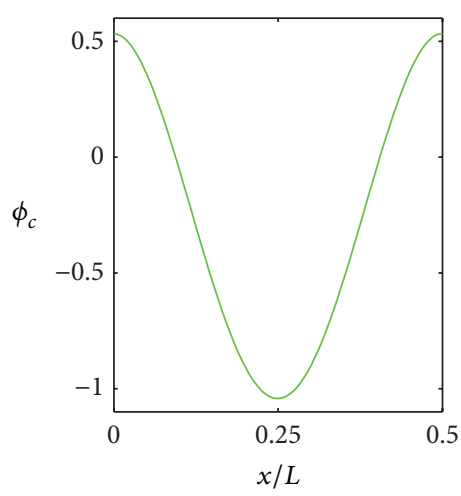

(c)

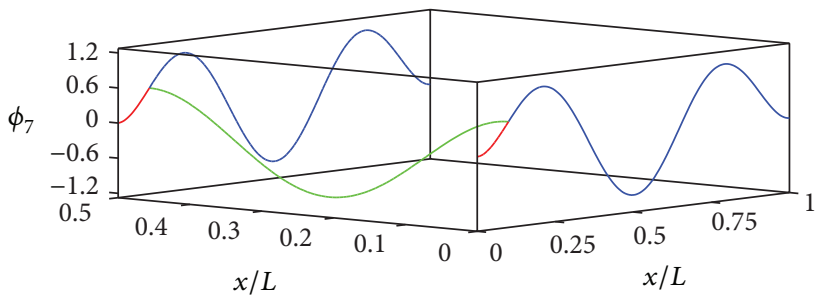

(d)

Figure 8: Seventh mode shape of the filter: (a) input, (b) output, (c) coupling beams, and (d) combination of (a), (b), and (c).

at the middle of the primary beams. As the coupling beam moves away from the middle to the other clamping points of the primary resonators, the first and second frequencies move closer to each other until they become equal to the fundamental natural frequency of a single resonator at the clamping points.
Variation of the center frequency $F_{0}$ of the filter is shown in Figure 13. We note that this variation is insensitive to the attachment location; it varies only $2.3 \mathrm{kHz}$ through the whole range. On the other hand, the bandwidth $\mathrm{BW}_{0}$ of the filter, shown in Figure 14, changes significantly as the coupling location sweeps the whole length of the primary 


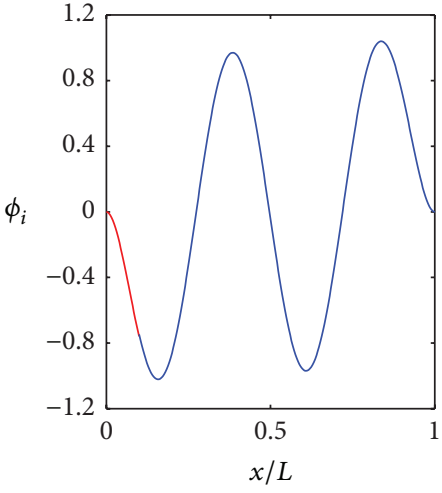

(a)

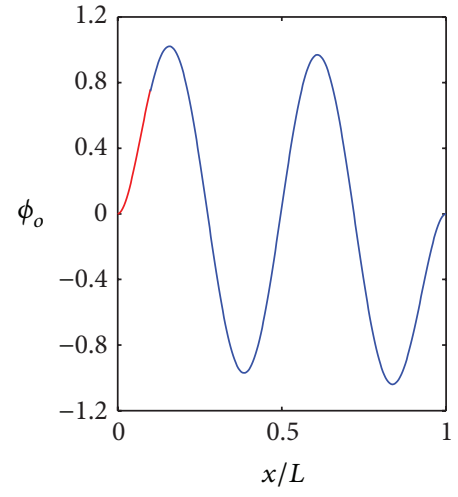

(b)

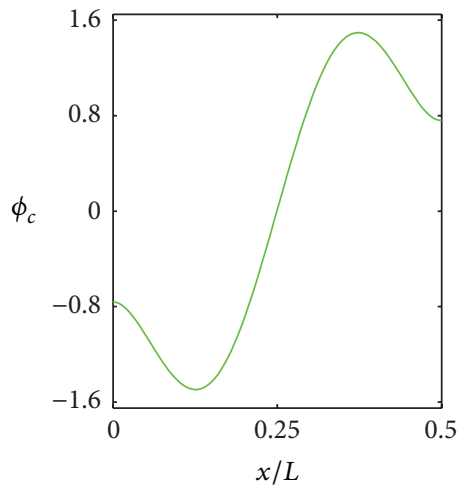

(c)

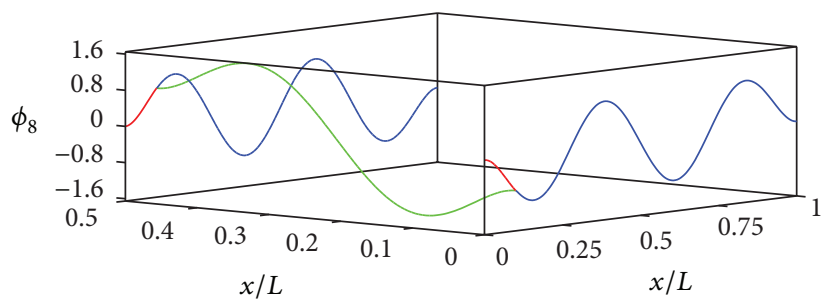

(d)

FigURE 9: Eighth mode shape of the filter: (a) input, (b) output, (c) coupling beams, and (d) combination of (a), (b), and (c).

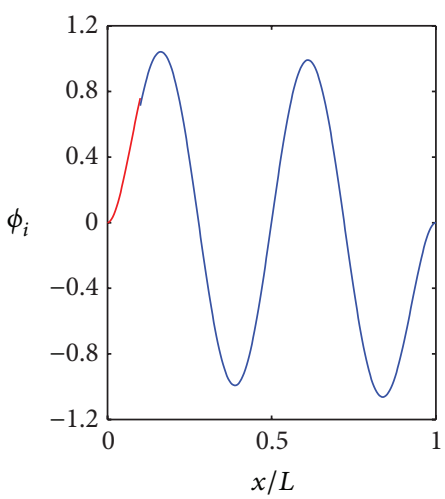

(a)

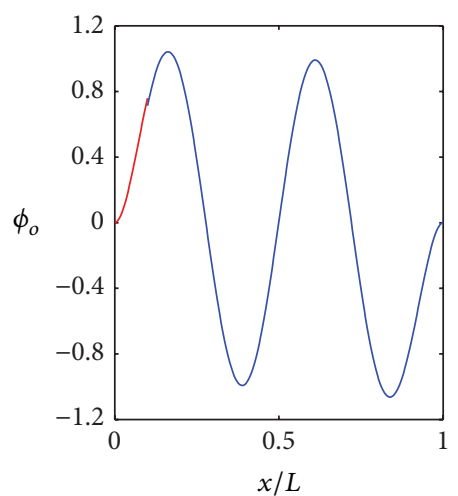

(b)

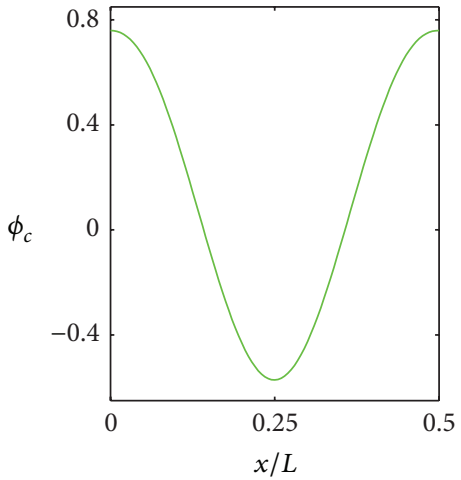

(c)

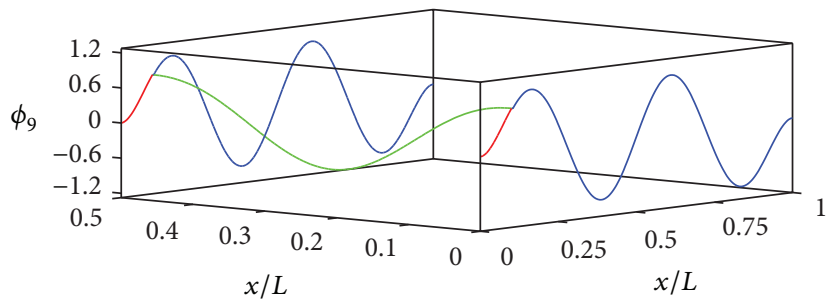

(d)

FIGURE 10: Ninth mode shape of the filter: (a) input, (b) output, (c) coupling beams, and (d) combination of (a), (b), and (c).

beams. The widest bandwidth is realized when the coupling beam is attached to the midpoints of the primary resonators, and it becomes narrower as the attachment location moves towards the clamping points. Because wireless systems transmit and receive signals within a narrow bandwidth allocated to the user in a communications environment crowded with interferers [12], attaching the coupling beam close to the clamping points is desirable because it yields a narrow bandwidth even though the center frequency is not a maximum there. 


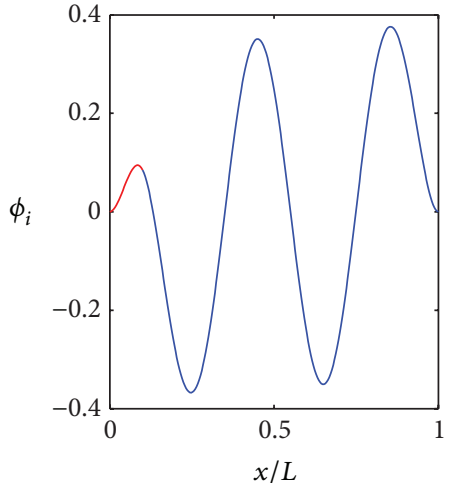

(a)

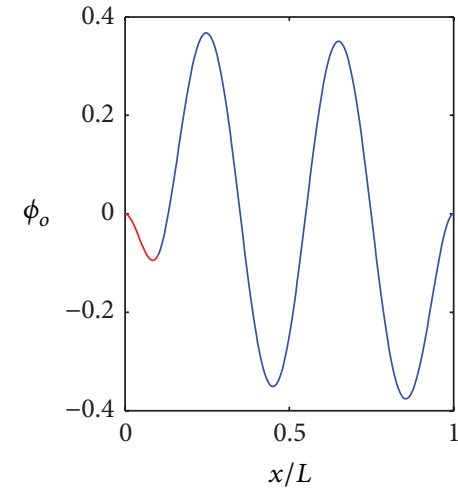

(b)

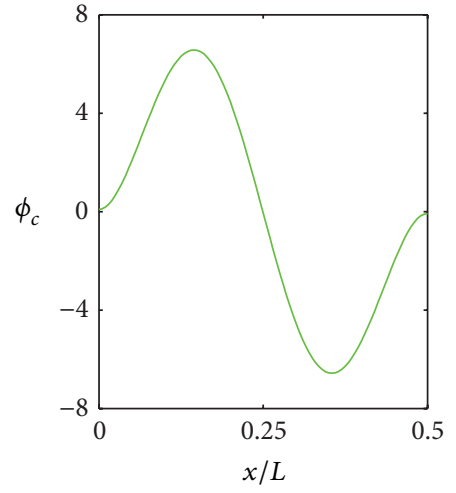

(c)

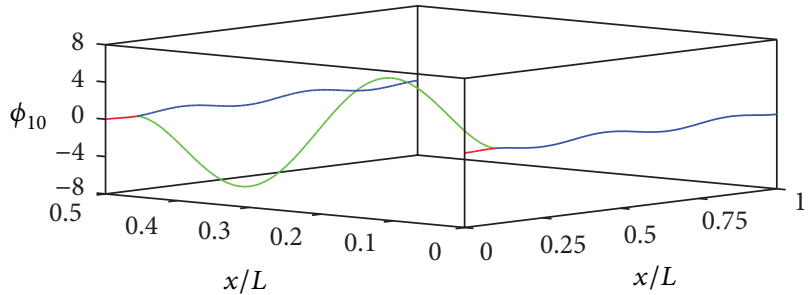

(d)

FIgURE 11: Tenth mode shape of the filter: (a) input, (b) output, (c) coupling beams, and (d) combination of (a), (b), and (c).

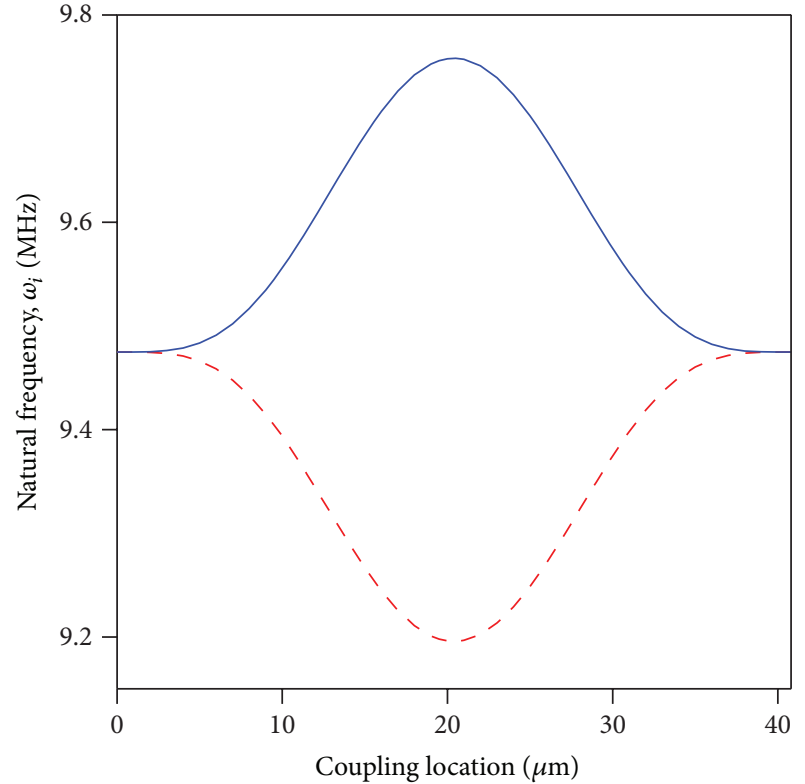

FIGURE 12: Variation of the first (dashed line) and second (solid line) natural frequencies of the filter with the attachment location.

The behavior of the bandwidth in Figure 14 is explained using the following equation $[2,9]$ :

$$
\mathrm{BW}_{0}=F_{0} \frac{k_{\mathrm{cs}}}{k_{\mathrm{cc}} k_{\mathrm{cl}}},
$$

where $k_{\mathrm{cs}}$ is the coupling spring constant, $k_{\mathrm{cc}}$ is the normalized coupling coefficient between the resonator tanks for

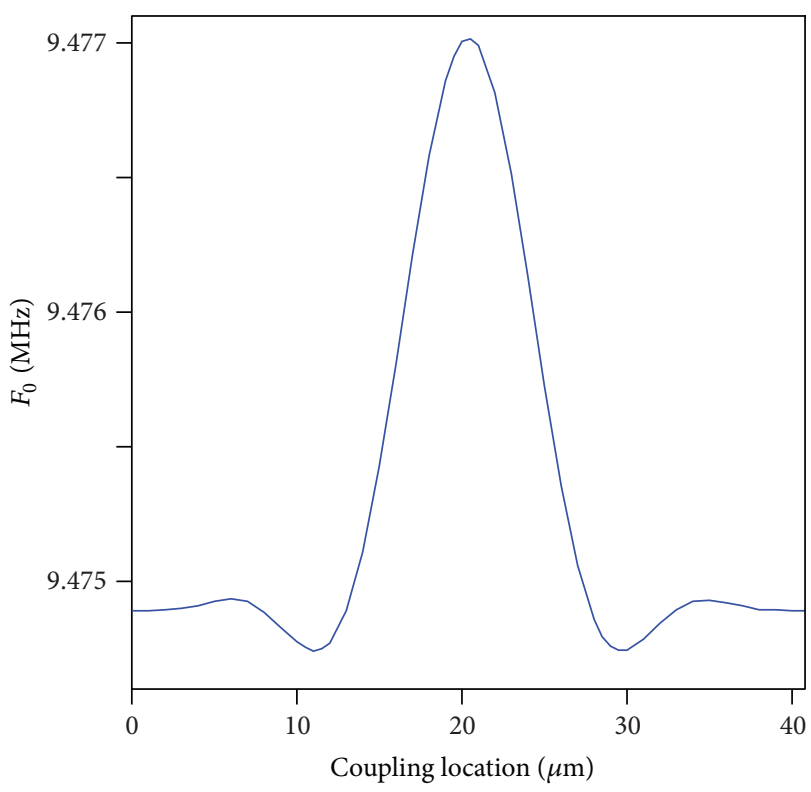

FIGURE 13: Variation of the center frequency of the unactuated filter with the attachment location.

a given filter type, and $k_{\mathrm{cl}}$ is the resonator stiffness at the coupling location. For a given type of filter and specified length and width of the coupling beam, the only important factor that determines the bandwidth is $k_{\mathrm{cl}}$. So that, for locations close to the clamping ends, the stiffness of the primary beam is high due to the shortness of the resonator, resulting in a narrow bandwidth. But when the attachment 


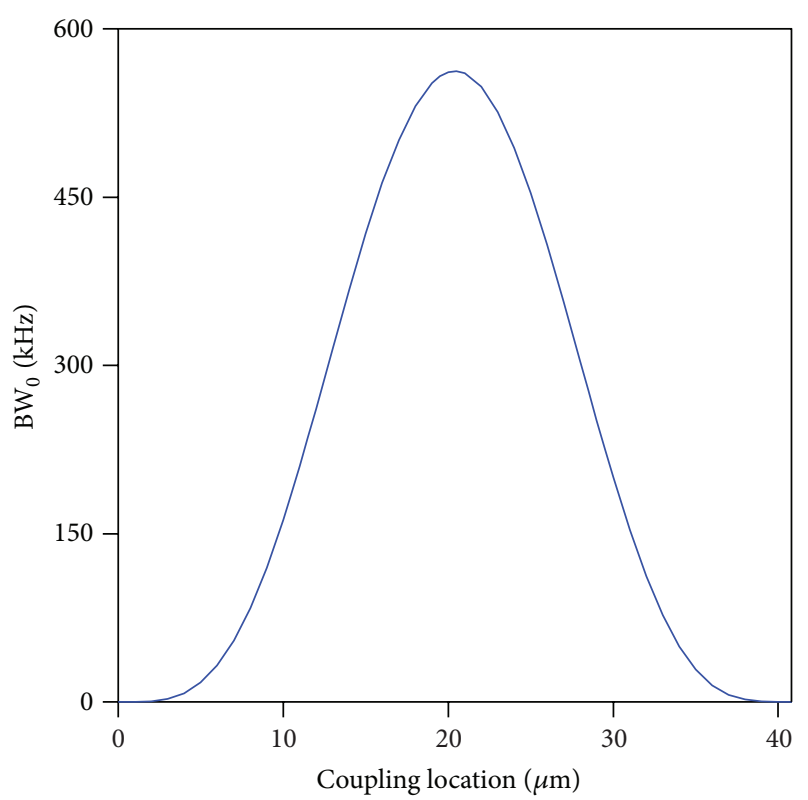

FIGURE 14: Variation of the bandwidth of the unactuated filter with the attachment location.

locations are at the midpoints of the primary resonators, the stiffness is the smallest (because the length has the largest value), leading to the widest bandwidth.

Based on Figures 13 and 14, we plot the quality factor $Q_{0, \text { filter }}$ of the filter using the following equation:

$$
Q_{0, \text { filter }}=\frac{F_{0}}{\mathrm{BW}_{0}}
$$

and show the result in Figure 15. It is clear that, as the attachment location moves closer to the middle of the primary beams, $Q_{0 \text {,filter }}$ becomes smaller and smaller and it is the highest close to the clamping points. Figures 14 and 15 are in qualitative agreement with the simulation results of the quality factor and bandwidth of the actual filter reported in [9].

It is important to mention that the actual filter center frequency, bandwidth, and quality factor are obtained when the filter is excited by proper electric signals applied to the primary resonators. However, the designer can obtain qualitative ideas about the effect of a variety of parameters on the filter performance from Figures 12-15 for unactuated filters. A quantitative description needs a high-level model in which the interaction between the electric force and the deflection of the beams is taken into consideration.

Next, we discuss the effect of the axial load on the first and second natural frequencies of the filter, and, to be more specific, on the center frequency $F_{0}$ and bandwidth $\mathrm{BW}_{0}$ of the unactuated filter. For the filter structure under study, because the primary beams are in direct contact with the substrate, the effect of temperature variation on these beams is much more than its effect on the coupling beam. So that, for the purpose of analysis and discussion here, we assume that the coupling beam is free of any stress and vary the axial stress in the primary beams. Increasing the tensile load (negative values of the axial load) shifts the center frequency of the unactuated filter $F_{0}$ to higher values, as shown in Figure 16. But in the case of increasing the compressive load (positive values of the axial load), the center frequency shifts to smaller values and eventually it reaches zero when the primary beams buckle. Moreover, increasing the tensile load produces a wider filter bandwidth $\mathrm{BW}_{0}$, as shown in Figure 17. But, more interestingly, as the compressive load increases, the bandwidth $\mathrm{BW}_{0}$ decreases slightly before it expands for large compressive loads.

We have assumed ideal clamped-clamped boundary conditions in this paper. In reality, these ends have finite rigidity and some structures have step-up type [13] or special design [9] supports. Consequently, the actual natural frequencies are lower than those of a microbeam with ideal clamped-clamped ends. Bannon et al. [9] and Wong [3] used a frequency modification factor obtained using the finite-element package to account for the difference between the natural frequencies of ideal clamped-clamped beams and those of the actual beams they have fabricated.

\section{Conclusions}

We solved the linear, undamped, and unforced vibration problem of micromechanical filters and obtained closedform expressions for their natural frequencies and mode shapes. The model described in this work treats the filter as a distributed-parameter system. For a micromechanical filter made of two clamped-clamped beam resonators connected via a coupling beam, we solved a boundary-value problem (BVP) composed of five equations and twenty boundary conditions for its natural frequencies and mode shapes. Instead of dealing with such a large system of equations, we suggested a method to reduce the problem to a set of three linear homogeneous algebraic equations for three constants and the frequencies. In addition to time and cost savings due to dealing with smaller system, the relation between the filter structure parameters became clearer. Setting the determinant of the reduced coefficient matrix equal to zero, we obtained the characteristic equation, which we solved for the natural frequencies. Then, we computed the constants and hence the mode shapes.

We compared our analytical procedure investigated in this paper with a numerical approach by using the finiteelement package ANSYS. The agreement was excellent validating our methodology. The closed-form expressions obtained in this work are easier to handle, more robust, and accurate. They are especially valuable in developing reduced-order models for the nonlinear static and dynamic characteristics of filters using the Galerkin procedure.

Due to the fact that the coupling beam is weak, the natural frequencies of the single resonator are split into two close frequencies for the filter: one frequency corresponds to an in-phase mode and the other corresponds to an outof-phase mode. Moreover, we discussed the effect of the attachment position on the bandwidth and quality factor of the unactuated filter and found that our results are in qualitative agreement with published results. We noted that 


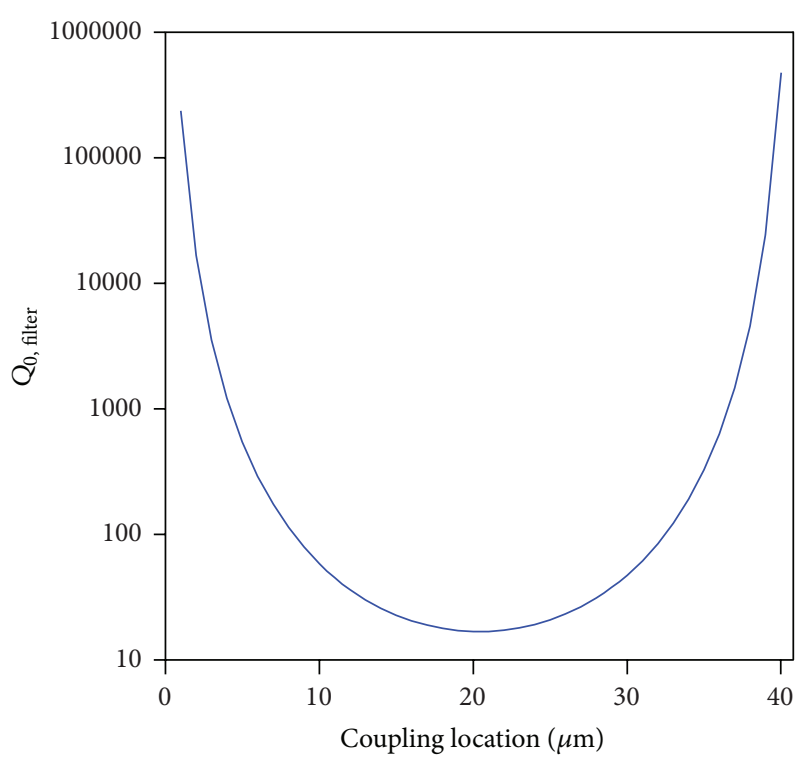

FIGURE 15: Variation of the quality factor of the unactuated filter with the attachment location.

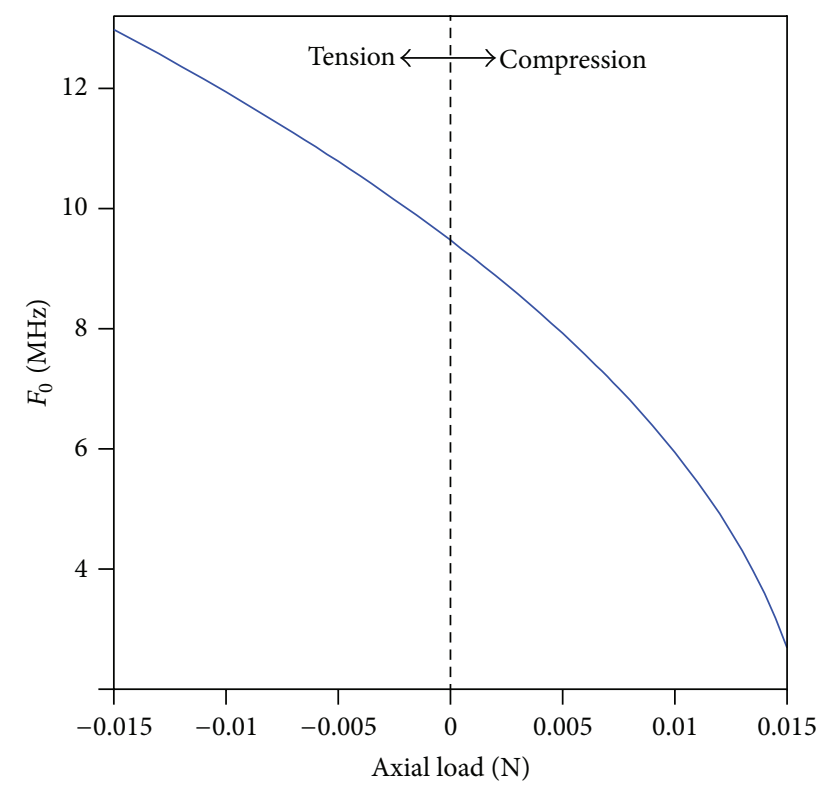

FIGURE 16: Variation of the center frequency of the unactuated filter with axial load in the primary beams.

the bandwidth is very sensitive to the coupling location, whereas the center frequency is not. Although the results in this paper are developed for an unactuated filter, they give the designer a qualitative idea about the effect of a variety of parameters on its performance. Actual filter specifications (center frequency, bandwidth, and quality factor) are obtained when electric signals are applied to the input and output resonators. The latter is the subject of other works $[6,14]$. In this work, we focused on the first two frequencies of the filter and discussed its center frequency and bandwidth because it is usually excited directly with a frequency in

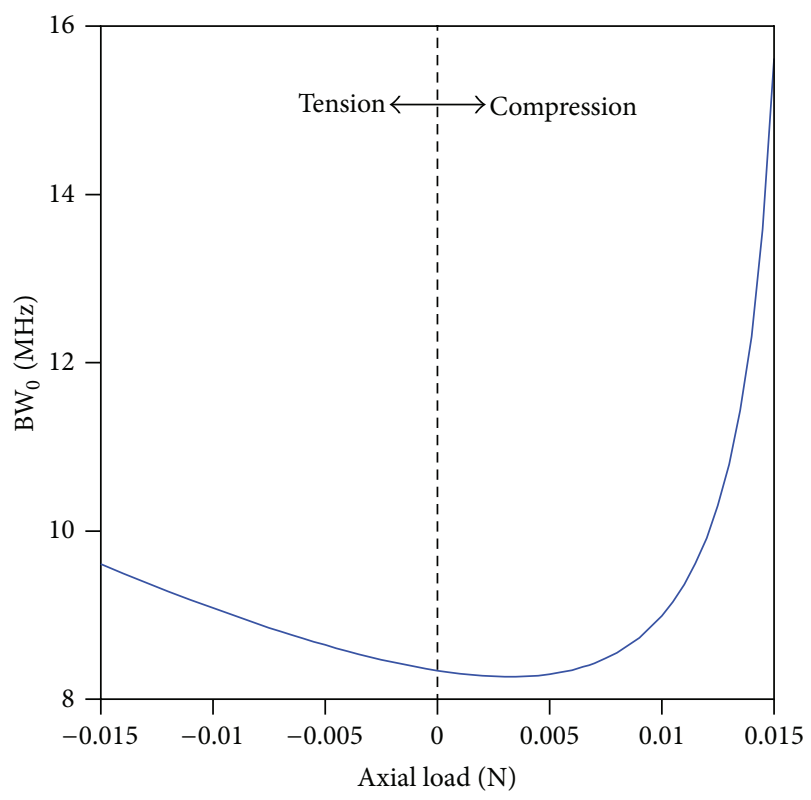

FIGURE 17: Variation of the bandwidth of the unactuated filter with axial load in the primary beams.

the neighborhood of these frequencies. In the case where higher modes are directly excited, the definition of the center frequency and bandwidth of the filter should be modified.

In addition, we studied the effect of axial loads applied to the beam resonators on the natural frequencies and bandwidth of the filter. More interestingly, we found that, as the compressive load increases from no axial load case, the bandwidth of the unactuated filter becomes narrower before it becomes larger for large compressive loads.

Finally, we emphasize that although the model presented in this paper for the vibration problem is restricted to micromechanical filters made of two clamped-clamped beam resonators connected via a coupling beam, they can be easily modified and adjusted to model any mechanically coupled microbeam arrays, such as, but not limited to, free-free microbeam array, higher-order filters, and arrays with bridges between nonadjacent resonators $[15,16]$.

\section{Conflict of Interests}

The author declares that there is no conflict of interests regarding the publication of this paper.

\section{References}

[1] V. B. Chivukula and J. F. Rhoads, "Microelectromechanical bandpass filters based on cyclic coupling architectures," Journal of Sound and Vibration, vol. 329, no. 20, pp. 4313-4332, 2010.

[2] K. Wang and C. T.-C. Nguyen, "High-order medium frequency micromechanical electronic filters," Journal of Microelectromechanical Systems, vol. 8, no. 4, pp. 534-557, 1999.

[3] A.-C. Wong, VHF microelectromechanical mixer-filters [Ph.D. thesis], University of Michigan, 2001. 
[4] M. U. Demirci, Micromechanical composite array resonators and filters for communications [Ph.D. thesis], University of Michigan, 2005.

[5] S.-S. Li, M. U. Demirci, Y.-W. Lin, Z. Ren, and C. T.-C. Nguyen, "Bridged micromechanical filters," in Proceedings of the IEEE International Ultrasonics, Ferroelectrics, and Frequency Control Joint 50 Anniversary Conference, pp. 280-286, Montreal, Canada, August 2004.

[6] B. K. Hammad, E. M. Abdel-Rahman, and A. H. Nayfeh, "Modeling and analysis of electrostatic MEMS filters," Nonlinear Dynamics, vol. 60, no. 3, pp. 385-401, 2010.

[7] A. Vyas and A. K. Bajaj, "Microresonators based on 1:2 internal resonance," in Proceedings of the ASME International Mechanical Engineering Congress and Exposition, Paper \# IMECE200581701, Orlando, Fla, USA, 2005.

[8] A. Vyas, D. Peroulis, and A. K. Bajaj, "Dynamics of a nonlinear microresonator based on resonantly interacting flexuraltorsional modes," Nonlinear Dynamics, vol. 54, no. 1-2, pp. 31-52, 2008.

[9] F. D. Bannon, J. R. Clark, and C. T.-C. Nguyen, "Nguyen high-Q HF microelectromechanical filters," IEEE Journal of Solid-State Circuits, vol. 35, no. 4, pp. 512-526, 2000.

[10] B. K. Hammad, E. M. Abdel-Rahman, and A. H. Nayfeh, "Characterization of a tunable MEMS RF filter," in Proceedings of the ASME International Mechanical Engineering Congress and Exposition, Paper \# IMECE2006-14136, Chicago, Ill, USA, 2006.

[11] L. Meirovitch, Fundamentals of Vibrations, McGraw-Hill, New York, NY, USA, 1st edition, 2001.

[12] H. J. de los Santos, RF MEMS Circuit Design for Wireless Communications, Artech House, Boston, Mass, USA, 1st edition, 2002.

[13] Q. Meng, M. Mehregany, and R. L. Mullen, "Theoretical modeling of microfabricated beams with elastically restrained supports," Journal of Microelectromechanical Systems, vol. 2, no. 3, pp. 128-137, 1993.

[14] B. K. Hammad, A. H. Nayfeh, and E. M. Abdel-Rahman, "A study of subharmonic excitation of mechanically coupled microbeams for filtration," in Proceedings of the ASME International Mechanical Engineering Congress and Exposition (IMECE '08), Paper \# IMECE2008-66728, pp. 371-384, Boston, Mass, USA, November 2008.

[15] S.-S. Li, M. U. Demirci, Y.-W. Lin, Z. Ren, and C. T.-C. Nguyen, "Bridged micromechanical filters," in Proceedings of the IEEE International Ultrasonics, Ferroelectrics, and Frequency Control Joint 50 Anniversary Conference, pp. 280-286, Montreal, Canada, 2004.

[16] S. S. Li, Y. -W. Lin, Z. Ren, and C. T.-C. Nguyen, "Self-switching vibrating micromechanical filter bank," in Proceedings of the Joint IEEE International Frequency Control/Precision Time and Time Interval Symposium, pp. 135-141, Vancouver, Canada, 2005. 

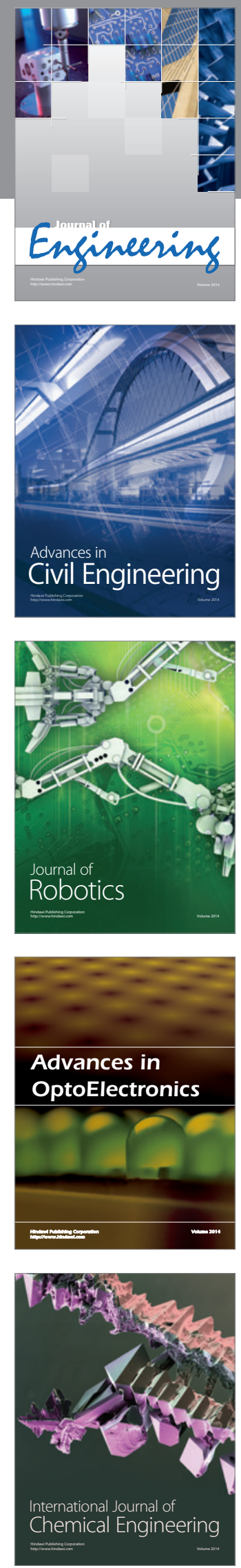

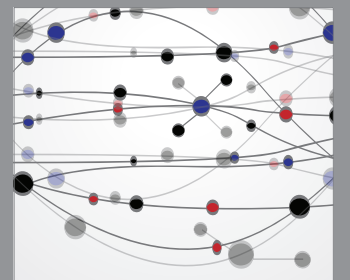

The Scientific World Journal
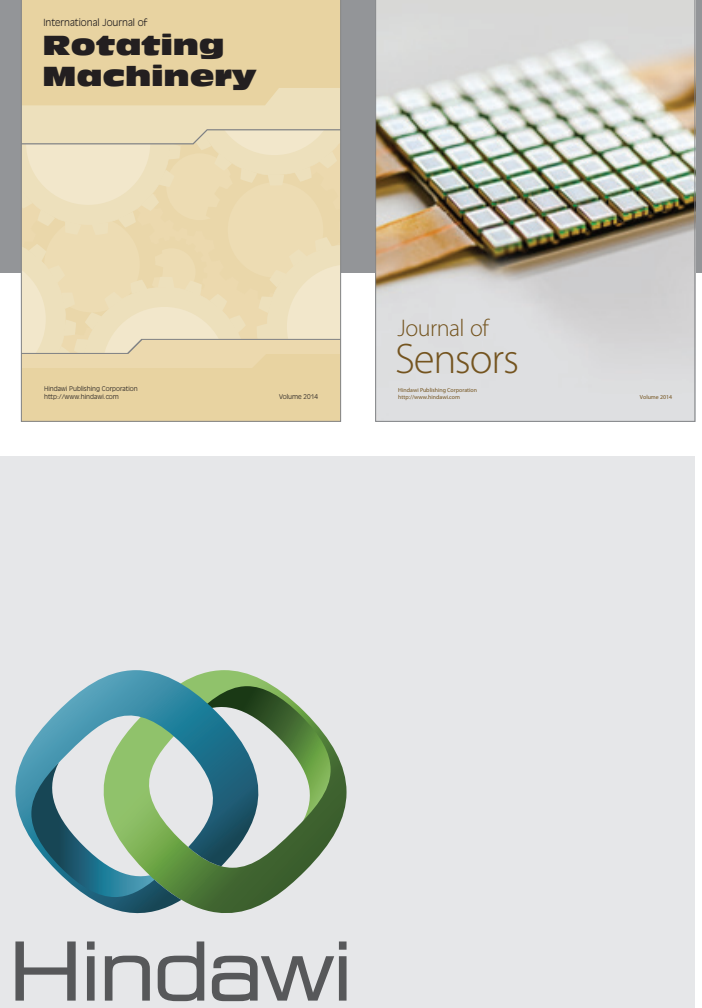

Submit your manuscripts at http://www.hindawi.com
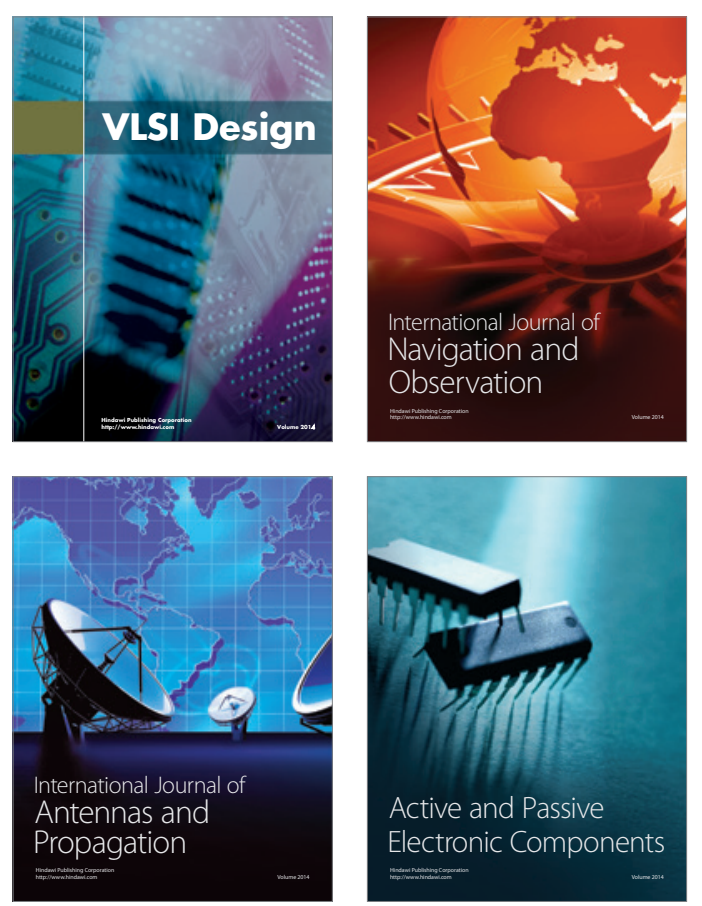
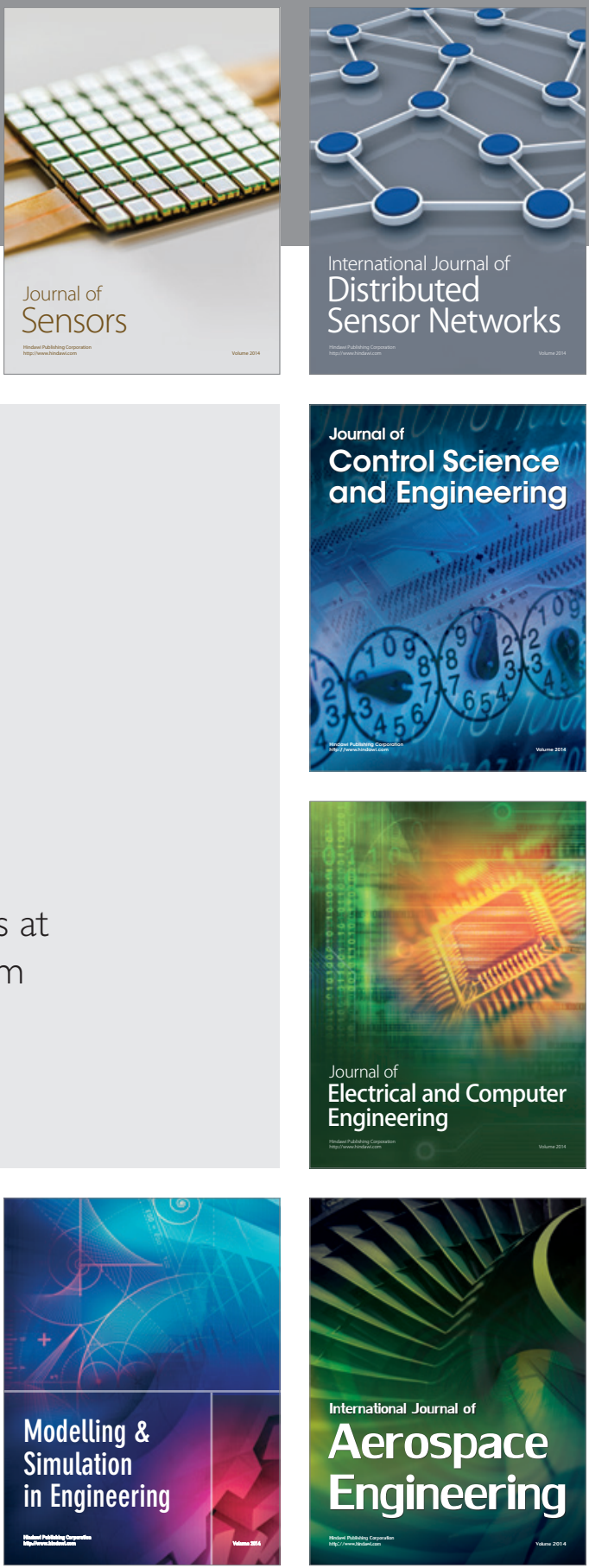

Journal of

Control Science

and Engineering
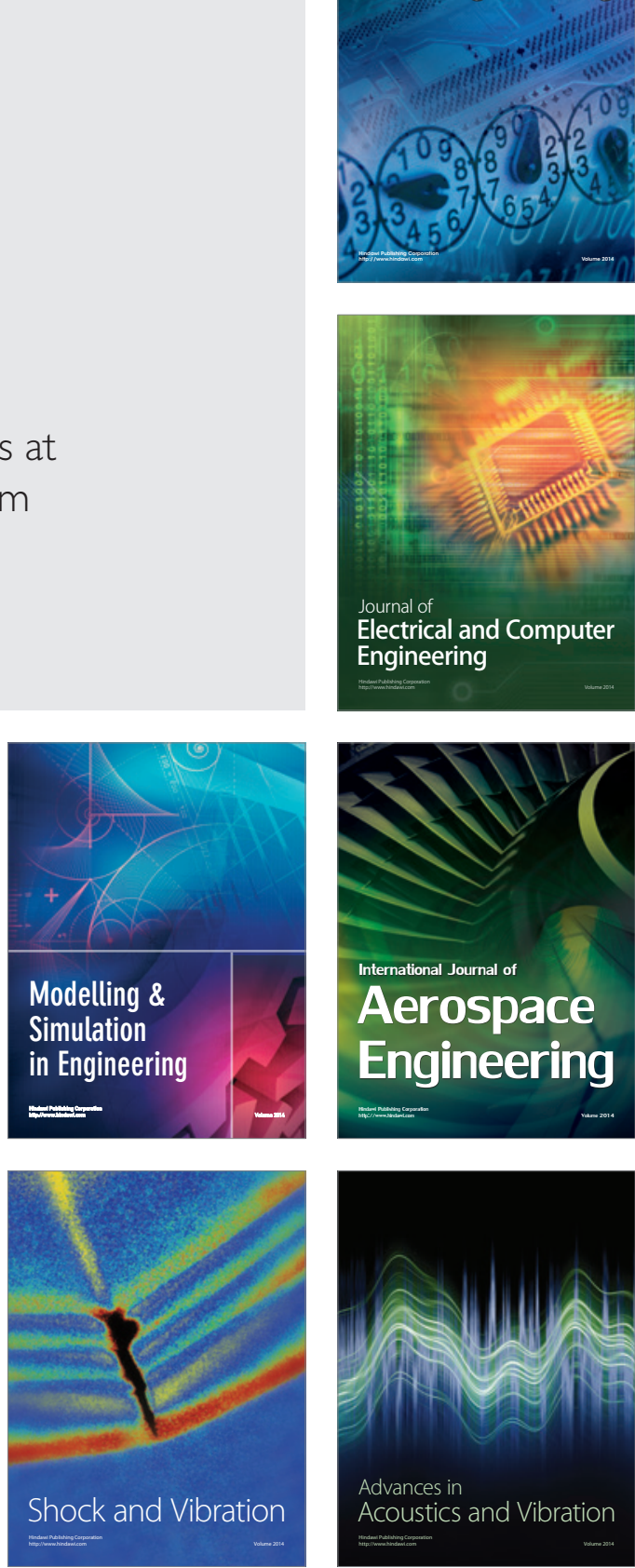